\title{
Almost PRIMES IN ALMOST ALL SHORT INTERVALS
}

\author{
JONI TERÄVÄINEN
}

\begin{abstract}
Let $E_{k}$ be the set of positive integers having exactly $k$ prime factors. We show that almost all intervals $\left[x, x+\log ^{1+\varepsilon} x\right]$ contain $E_{3}$ numbers, and almost all intervals $\left[x, x+\log ^{3.51} x\right]$ contain $E_{2}$ numbers. By this we mean that there are only $o(X)$ integers $1 \leq x \leq X$ for which the mentioned intervals do not contain such numbers. The result for $E_{3}$ numbers is optimal up to the $\varepsilon$ in the exponent. The theorem on $E_{2}$ numbers improves a result of Harman, which had the exponent $7+\varepsilon$ in place of 3.51. We also consider general $E_{k}$ numbers, and find them on intervals whose lengths approach $\log x$ as $k \rightarrow \infty$.
\end{abstract}

\section{INTRODUCTION}

When studying $E_{k}$ numbers (products of exactly $k$ primes), it is natural to ask, how short intervals include such numbers almost always. Since Wolke's work [21, the essential question has been minimizing the number $c$ such that almost all intervals $\left[x, x+\log ^{c} x\right]$ contain an $E_{k}$ number, meaning that all but $o(X)$ such intervals with integer $x \in[1, X]$ contain such a number. Wolke showed in 1979 that the value $c=5 \cdot 10^{6}$ is admissible for $E_{2}$ numbers. This was improved to $c=7+\varepsilon$ for $E_{2}$ numbers by Harman [9] in 1982. Wolke's and Harman's methods are based on reducing the problem to estimates for sums over the zeros of the Riemann zeta function, and on the fact that the density hypothesis is known to hold in a non-trivial strip (namely Jutila's [14] region $\sigma \geq \frac{11}{14}$ in Harman's argument ${ }^{1}$ ). To the author's knowledge, Harman's exponent for $E_{2}$ numbers was the best one known also for $E_{k}$ numbers with $k \geq 3$.

If one considers $P_{k}$ numbers, which are products of no more than $k$ primes, one can obtain improvements. Mikawa [16] showed in 1989 that for any function $\psi(x)$ tending to infinity, the interval $\left[x, x+\psi(x) \log ^{5} x\right]$ contains a $P_{2}$ number almost always. Furthermore, Friedlander and Iwaniec [4, Chapters 6 and 11] proved that for any such function $\psi(x)$ the interval $[x, x+\psi(x) \log x]$ contains a $P_{4}$ number almost always. They also hint how to prove the same result for $P_{3}$ numbers. There is however a crucial difference between $E_{k}$ and $P_{k}$ numbers, since the $E_{k}$ numbers are subject to the famous parity problem, and hence cannot be dealt with using only classical combinatorial sieves, which are the basis of the arguments on $P_{k}$ numbers. Therefore, the $E_{k}$ numbers are also a much closer analog of primes than the $P_{k}$ numbers.

\footnotetext{
${ }^{1}$ In fact, introducing into Harman's argument the widest known den'sity hypothesis region $\sigma \geq \frac{25}{32}$, due to Bourgain [2] from 2000, would give $c=6.86$.
} 
One would naturally expect almost all intervals $[x, x+\psi(x) \log x]$ to have also prime numbers in them, and this would follow from the heuristic that the proportion of $x$ for which $[x, x+\lambda \log x]$ contains exactly $m$ primes for fixed $m$ and $\lambda>0$ should be given by the Poisson distribution $\frac{\lambda^{m}}{m !} e^{-\lambda}$. Such results are however far beyond the current knowledge, as the shortest intervals, almost all of which are known to contain primes, are $\left[x, x+x^{\frac{1}{20}+\varepsilon}\right]$ by a result of Jia [13]. However, the results of Goldston-Pintz-Yıldırım [6], [7] on short gaps between primes tell that for any $\lambda>0$ there is a positive proportion of integers $x \leq X$ for which $[x, x+\lambda \log x]$ contains a prime, but it is not known whether this proportion approaches 1 as $\lambda$ increases. A recent result of Freiberg [3], in turn, gives exactly $m$ primes on an interval $[x, x+\lambda \log x]$ for at least $X^{1-o(1)}$ integers $x \leq X$. Concerning conditional results, Gallagher [5] showed that the Poisson distribution of primes in short intervals would follow from a certain uniform form of the Hardy-Littlewood prime $k$-tuple conjecture. Under the Riemann hypothesis, it was shown by Selberg [18] in 1943 that almost all intervals $\left[x, x+\psi(x) \log ^{2} x\right]$ contain primes. For $E_{2}$ numbers, under the density hypothesis, Harman's argument from [9] would give the exponent $c=3+\varepsilon$.

In this paper, we establish the exponent $c=1+\varepsilon$ for $E_{3}$ numbers and the exponent $c=3.51$ for $E_{2}$ numbers. Our results for $E_{2}, E_{3}$ and $E_{k}$ numbers are stated as follows.

Theorem 1. Almost all intervals $\left[x, x+(\log \log x)^{6+\varepsilon} \log x\right]$ contain a product of exactly three distinct primes.

Theorem 2. For any integer $k \geq 4$, there exists $C_{k}>0$ such that almost all intervals $\left[x, x+\left(\log _{k-1} x\right)^{C_{k}} \log x\right]$ contain a product of exactly $k$ distinct primes. Here $\log _{\ell}$ is the eth iterated logarithm.

Theorem 3. Almost all intervals $\left[x, x+\log ^{3.51} x\right]$ with $x \leq X$ contain a product of exactly two distinct primes.

Theorems 1 and 2 are direct consequences of the following theorem.

Theorem 4. Let $X$ be large enough, $k \geq 3$ a fixed integer, and $\varepsilon>0$ small enough but fixed. Define the numbers $P_{1}, \ldots, P_{k-1}$ by setting $P_{k-1}=(\log X)^{\varepsilon^{-2}}, P_{k-2}=(\log \log X)^{6+10 \sqrt{\varepsilon}}$ and $P_{j}=\left(\log P_{j+1}\right)^{\varepsilon^{-1}}$ for $1 \leq j \leq k-3$. For $P_{1} \log X \leq h \leq X$, we have

$$
\left|\frac{1}{h} \sum_{\substack{x \leq p_{1} \cdots p_{k} \leq x+h \\ P_{i} \leq p_{i} \leq P_{i}^{1+\varepsilon}, i \leq k-1}} 1-\frac{1}{X} \sum_{\substack{X \leq p_{1} \cdots p_{k} \leq 2 X \\ P_{i} \leq p_{i} \leq P_{i}^{1+\varepsilon}, i \leq k-1}} 1\right| \ll \frac{1}{(\log X)\left(\log _{k} X\right)}
$$

for almost all $x \leq X$.

In the theorem above, the average over the dyadic interval is $\gg \frac{1}{\log X}$ by the prime number theorem, so Theorems 1 and 2 indeed follow from Theorem 4 . Similarly, Theorem 3 is a direct consequence of the following. 
Theorem 5. Let $X$ be large enough, $P_{1}=\log ^{a} X$ with $a=2.51, \varepsilon>0$ fixed, and $P_{1} \log X \leq h \leq X$. We have

$$
\frac{1}{h} \sum_{\substack{x \leq p_{1} p_{2} \leq x+h \\ P_{1} \leq p_{1}<P_{1}^{1+\varepsilon}}} 1 \gg \frac{1}{X} \sum_{\substack{X \leq p_{1} p_{2} \leq 2 X \\ P_{1} \leq p_{1} \leq P_{1}^{1+\varepsilon}}} 1
$$

for almost all $x \leq X$.

Remark 1. Since $h \geq P_{1} \log X$, we have the dependence $c=a+1$ between the exponent $a$ in Theorem 5 and the smallest exponent $c$ for which we can show that the interval $\left[x, x+\log ^{c} x\right]$ contains an $E_{2}$ number almost always.

Remark 2. Note that Theorems 4 and 5 tell us that there are $\gg \frac{h}{\log X} E_{k}$ numbers in almost all intervals $[x, x+h]$, where $h$ and $k$ are as in one of the theorems. However, we are not quite able to find $E_{k}$ numbers on intervals $[x, x+\psi(x) \log x]$ with $\psi$ tending to infinity arbitrarily slowly, unlike in the result of Friedlander and Iwaniec on $P_{k}$ numbers. In addition, our bound for the number of exceptional values is at best $\ll \frac{x}{\log ^{\varepsilon} x}$ and often weaker, while the methods used in [10], [13] and [20] for primes in almost all short intervals have a tendency to give the bound $\ll \frac{x}{\log ^{A} x}$ for any $A>0$, when they work. The limit of our method for $E_{2}$ numbers is the exponent $3+\varepsilon$, as will be seen later, so proving for example unconditionally the analog of Selberg's result for $E_{2}$ numbers would require some further ideas.

To prove our results, we adapt the ideas of the paper [15] of Matomäki and Radziwilł on multiplicative functions in short intervals to considering almost primes in short intervals. In that paper, a groundbreaking result is that for any multiplicative function, with values in $[-1,1]$, its average over $[x, x+h]$ is almost always asymptotically equal to its dyadic average over $[x, 2 x]$, with $h=h(x) \leq x$ any function tending to infinity. The error terms obtained there for general multiplicative functions are not quite good enough for our purposes. Nevertheless, using similar techniques, and replacing the multiplicative function with the indicator function of the numbers $p_{1} \cdots p_{k}$, with $p_{i}$ primes from carefully chosen intervals, allows us to find $E_{k}$ numbers on intervals $[x, x+h]$, with $\frac{h}{\log x}$ growing very slowly. In this setting, we can apply various mean, large and pointwise value results for Dirichlet polynomials, some of which work specifically with primes or the zeta function, but not with general multiplicative functions (such as Watt's theorem on the twisted moment of the Riemann zeta function, a large values theorem from [15] for Dirichlet polynomials supported on primes, and Vinogradov's zero-free region). In many places in the argument, we cannot afford to lose even factors of $\log ^{\varepsilon} x$, so we need to factorize Dirichlet polynomials in a manner that is nearly nearly lossless, and use an improved form of the mean value theorem for Dirichlet polynomials. To deal with some of the arising Dirichlet polynomials, we also need some sieve methods, similar to those that have been successfully applied to finding primes in short intervals for example in [10], [13] and [20]. In the case of $E_{2}$ numbers, in addition to these methods, we benefit from the theory of exponent pairs and Jutila's large values theorem.

The structure of the proofs of Theorems 4 and 5 is the following. We will first present the lemmas necessary for proving Theorem 4 , and hence Theorems 1 and 2 . Besides employing 
these lemmas to prove Theorem 4, we notice that they are already sufficient for finding products of exactly two primes in almost all intervals $\left[x, x+\log ^{5+\varepsilon} x\right]$, which is as good as Mikawa's result for $P_{2}$ numbers up to $\varepsilon$ in the exponent (one could also get $c$ slightly below 5 using exponent pairs, which are just one of the additional ideas required for Theorem 5). The rest of the paper is then concerned with reducing the exponent $5+\varepsilon$ to 3.51 for products of two primes, and this requires some further ingredients, as well as all the lemmas that were needed for products of three or more primes.

\subsection{Acknowledgements}

The author is grateful to his supervisor Kaisa Matomäki for various useful comments and discussions. The author thanks the referee for careful reading of the paper and for useful comments. While working on this project, the author was supported by the Vilho, Yrjö and Kalle Vaisälä foundation of the Finnish Academy of Science and Letters.

\subsection{Notation}

The symbols $p, q, p_{i}$ and $q_{i}$ are reserved for primes, and $d, k, \ell, m$ and $n$ are always positive integers. We often use the same capital letter for a Dirichlet polynomial and its length. We call zeta sums partial sums of $\zeta(s)$ or $\zeta^{\prime}(s)$ of the form $\sum_{n \sim N} n^{-s}$ or $\sum_{n \sim N}(\log n) n^{-s}$.

The function $\nu(\cdot)$ counts the number of distinct prime divisors of a number, $\mu(\cdot)$ is the Möbius function, $\Lambda(\cdot)$ is the von Mangoldt function, and $d_{r}(m)$ is the number of solutions to $a_{1} \cdots a_{r}=m$ in positive integers. The function $\omega(\cdot)$ is Buchstab's function (see Harman's book [10, Chapter 1]), defined as $\omega(u)=\frac{1}{u}$ for $1 \leq u \leq 2$ and via the differential equation $\frac{d}{d u}(u \omega(u))=\omega(u-1)$ for $u>2$, imposing the requirement that $\omega$ be continuous on $[1, \infty)$. We make the convention that $\omega(u)=0$ for $u<1$. In addition, let $\mathcal{P}(z)=\prod_{p<z} p$, and let $S(A, \mathbb{P}, z)$ count the numbers in $A$ coprime to $\mathcal{P}(z)$.

The quantity $\varepsilon>0$ is always small enough but fixed. The symbols $C_{1}, C_{2}, \ldots$ denote unspecified, positive, absolute constants. By writing $n \sim X$ in a summation, we mean $X \leq n<2 X$. The expression $1_{S}$ is the indicator function of the set $S$, so that $1_{S}(n)=1$ if $n \in S$ and $1_{S}(n)=0$ otherwise. We use the usual Landau and Vinogradov asymptotic notation $o(\cdot), O(\cdot)$ and $\ll, \gg$. The notation $X \asymp Y$ is shorthand for $X \ll Y \ll X$.

\section{PRELIMINARY LEMMAS}

\subsection{Reduction to mean values of Dirichlet polynomials}

We present several lemmas that are required for proving both Theorems 4 and 5 , Later on, we give some additional lemmas that are needed only for proving Theorem 5 .

The plan of the proofs of Theorems 4 and 5, and hence of Theorems 1 , 2 and 3 , is to transform the problem of comparing almost primes in short and long intervals to finding cancellation in the mean square of the corresponding Dirichlet polynomial. The polynomial can be factorized after it is divided into short intervals, and different methods can 
be applied to different factors. This approach is utilized in many earlier works on primes and almost primes in short intervals; see e.g. [10, [15]. We then apply several mean, large and pointwise value theorems, which are presented in Subsection 2.3, to find the desired cancellation in the Dirichlet polynomial.

The following Parseval-type lemma allows us to reduce the problem of finding almost primes in short intervals to finding cancellation in a Dirichlet polynomial.

Lemma 1. Let

$$
S_{h}(x)=\frac{1}{h} \sum_{x \leq n \leq x+h} a_{n}
$$

where $a_{n}$ are complex numbers, and let $2 \leq h_{1} \leq h_{2} \leq \frac{X}{T_{0}^{3}}$ with $T_{0} \geq 1$. Also let $F(s)=$ $\sum_{n \sim X} \frac{a_{n}}{n^{s}}$. Then

$$
\begin{aligned}
\frac{1}{X} \int_{X}^{2 X}\left|\frac{1}{h_{1}} S_{h_{1}}(x)-\frac{1}{h_{2}} S_{h_{2}}(x)\right|^{2} d & \ll \frac{1}{T_{0}}+\int_{T_{0}}^{\frac{X}{h_{1}}}|F(1+i t)|^{2} d t \\
& +\max _{T \geq \frac{X}{h_{1}}} \frac{X}{T h_{1}} \int_{T}^{2 T}|F(1+i t)|^{2} d t .
\end{aligned}
$$

Proof. This is Lemma 14 in the paper [15] (except that we do not specify the value of $T_{0}$ ). A related bound can be found for example in [10, Chapter 9].

We choose $T_{0}=X^{0.01}$, and $h_{2}=\frac{X}{T_{0}^{3}}$ in Lemma 1 , and the average function $S_{h}(x)$ is given by the short average in (1) or (2). Now, defining

$$
F(s)=\sum_{\substack{p_{1} \cdots p_{k} \sim X \\ P_{i} \leq p_{i} \leq P_{i}^{1+\varepsilon}, i \leq k-1}}\left(p_{1} \cdots p_{k}\right)^{-s},
$$

where $P_{i}$ are as in Theorem 4 or 5 , proving Theorems 4 and 5 is reduced to showing that

$$
\int_{T_{0}}^{T}|F(1+i t)|^{2} d t=o\left(\left(\frac{T h}{X}+1\right) \cdot \frac{1}{\left(\log ^{2} X\right)\left(\log _{k} X\right)^{2}}\right),
$$

for $T_{0}=X^{0.01}$ and $h \geq P_{1} \log X$. Indeed, substituting this to Lemma 1 shows that

$$
\frac{1}{X} \int_{X}^{2 X}\left|\frac{1}{h} S_{h}(x)-\frac{1}{h_{2}} S_{h_{2}}(x)\right|^{2} d x=o\left(\frac{1}{\left(\log ^{2} X\right)\left(\log _{k} X\right)^{2}}\right),
$$

where $h_{2}=\frac{X}{T_{0}^{3}}$. It actually suffices to prove (4) for $T \leq X$, since otherwise the mean value theorem (Lemma 3) gives a good enough bound for the last term in (3).

Note that for $T \leq X$ the trivial bound for the integral in (4), coming from the mean value theorem, is $\ll(\log X)^{-1}$. Thus our task is to save slightly more than one additional 
logarithm in this integral (for $T \leq \frac{X}{h}$, at least).

Once the required estimates for Dirichlet polynomials have been established, we can apply the prime number theorem in short intervals with Vinogradov's error term (see [12, Chapter 10]) to see that

$$
\frac{1}{h_{2}} S_{h_{2}}(x)-\frac{1}{X} S_{X}(X) \ll \exp \left(-(\log X)^{\frac{3}{5}-\varepsilon}\right),
$$

for $h_{2}=x^{0.97}, x \sim X$, and hence deduce Theorems 4 and 5 (and consequently 1,2 and 3 ). For example, we compute

$$
\begin{aligned}
& \frac{1}{h_{2}} \sum_{\substack{x \leq p_{1} p_{2} p_{3} \leq x+h_{2} \\
P_{1} \leq p_{1} \leq P_{1}^{1+\varepsilon} \\
P_{2} \leq p_{2} \leq P_{2}^{1+\varepsilon}}}=\frac{1}{h_{2}} \sum_{\substack{P_{1} \leq p_{1} \leq P_{1}^{1+\varepsilon} \\
P_{2} \leq p_{2} \leq P_{2}^{1+\varepsilon}}}\left(\pi\left(\frac{x+h_{2}}{p_{1} p_{2}}\right)-\pi\left(\frac{x}{p_{1} p_{2}}\right)\right) \\
&=\frac{1}{h_{2}} \sum_{\substack{P_{1} \leq p_{1} \leq P_{1}^{1+\varepsilon} \\
P_{2} \leq p_{2} \leq P_{2}^{1+\varepsilon}}} \frac{h_{2}}{p_{1} p_{2} \log \frac{x}{p_{1} p_{2}}} \\
&+ O\left(\exp \left(-(\log x)^{\frac{3}{5}-\frac{\varepsilon}{2}}\right)\right) \\
&= \sum_{\substack{P_{1} \leq p_{1} \leq P_{1}^{1+\varepsilon} \\
P_{2} \leq p_{2} \leq P_{2}^{1+\varepsilon}}} \frac{1}{p_{1} p_{2} \log \frac{X}{p_{1} p_{2}}}+O\left(\exp \left(-(\log X)^{\frac{3}{5}-\varepsilon}\right)\right),
\end{aligned}
$$

and the same asymptotics hold for the dyadic sum. Sometimes we end up comparing the sums $\frac{1}{h_{2}} S_{h_{2}}(x)$ and $\frac{1}{x} S_{2}(x)$ with $a_{n}$ not quite equal to the coefficients of $F(s)$, but equal to the indicator function of the numbers $p_{1} p_{2} n$ with $p_{1}$ and $p_{2}$ from the intervals $\left[P_{1}, P_{1}^{1+\varepsilon}\right]$ and $\left[P_{2}, P_{2}^{1+\varepsilon}\right]$, respectively, and $n$ having no prime factors smaller than $p_{2}$. There may also be a simple cross-conditions on $p_{1}$ and $p_{2}$, but comparing the sums still causes no difficulty.

Thus, in the rest of the paper we can concentrate on bounding Dirichlet polynomials. Although there is a close analogy in the formulations of Theorems 4 and 5, estimating the polynomial arising from the latter is more difficult, and will require several additional ideas.

\subsection{Factorizations for Dirichlet polynomials}

In bounding Dirichlet polynomials, factorizations play an important role. We encounter situations where the only cross-condition on the variables in the polynomial is that their product belongs to a certain range, so the variables can be separated by diving them into short ranges and estimating the mean values of the resulting polynomials. The factorization is provided by the following lemma, which also takes into account the improved mean value theorem (Lemma 4). 
Lemma 2. Let $\mathcal{S} \subset[-T, T]$ be measurable and

$$
F(s)=\sum_{\substack{m n \sim X \\ M \leq m \leq M^{\prime}}} \frac{a_{m} b_{n}}{(m n)^{s}}
$$

for some $M^{\prime}>M \geq 2$ and for some complex numbers $a_{m}, b_{n}$. Let $H \geq 1$ be such that $H \log M$ and $H \log M^{\prime}$ are integers. Denote

$$
A_{v, H}(s)=\sum_{e^{\frac{v}{H}} \leq m<e^{\frac{v+1}{H}}} \frac{a_{m}}{m^{s}}, \quad B_{v, H}(s)=\sum_{n \sim X e^{-\frac{v}{H}}} \frac{b_{n}}{n^{s}} .
$$

Then

$$
\begin{aligned}
& \int_{\mathcal{S}}|F(1+i t)|^{2} d t \ll|I|^{2} \int_{\mathcal{S}}\left|A_{v_{0}, H}(1+i t) B_{v_{0}, H}(1+i t)\right|^{2} d t
\end{aligned}
$$

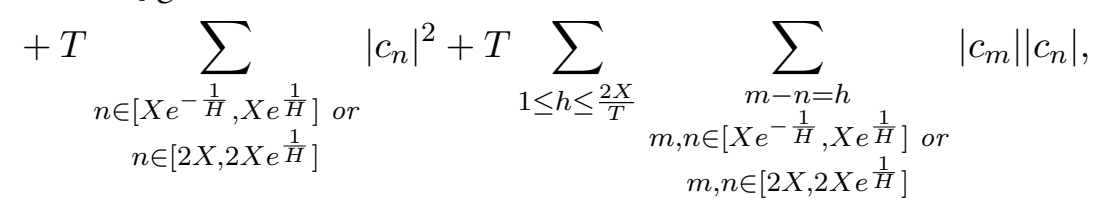

with

$$
c_{n}=\frac{1}{n} \sum_{\substack{n=k \ell \\ M \leq k \leq M^{\prime}}}\left|a_{k} b_{\ell}\right|,
$$

$I=\left[H \log M, H \log M^{\prime}\right)$ and $v_{0} \in I$ a suitable integer.

Remark 3. In applications we have $M^{\prime} \geq 2 M$, so the conditions that $H \log M$ and $H \log M^{\prime}$ be integers can be ignored, since we can always afford to vary $H$ and $M^{\prime}$ by the necessary amount.

Remark 4. When proving Theorem 4, we cannot afford to lose any powers of logarithm in some factorizations, and indeed the second term in the lemma crucially has the factor $T$ instead of the factor $X$ occurring in the mean value theorem, and in the first term we will lose a factor of size $\ll H^{2} \log ^{2} \frac{M^{\prime}}{M}$, which in practice is minuscule.

Proof. This resembles Lemma 12 in the paper [15] by Matomäki and Radziwiłł (where, in addition to factorization in short intervals, a Ramaré-type identity is used). We split $F(s)$ into short intervals, obtaining



Observe that $X e^{-\frac{v+1}{H}} \leq n<X e^{-\frac{v}{H}}$ can hold above only for $m n \in\left[X e^{-\frac{1}{H}}, X e^{\frac{1}{H}}\right]$. Furthermore, we always have $m n \in\left[X e^{-\frac{1}{H}}, 2 X e^{\frac{1}{H}}\right]$. This allows us to write

$$
F(s)=\sum_{v \in I \cap \mathbb{Z}} A_{v, H}(s) B_{v, H}(s)+\sum_{\substack{k \in\left[X e^{-\frac{1}{H}}, X e^{\frac{1}{H}}\right] o r \\ k \in\left[2 X, 2 X e \frac{1}{H}\right]}} \frac{d_{k}}{k^{s}}
$$


with

$$
\left|d_{k}\right| \leq \sum_{k=m n}\left|a_{m} b_{n}\right|
$$

Now the claim of the lemma follows by taking mean squares on both sides of (5) on the line $\Re(s)=1$, applying the improved mean value theorem (Lemma 4), and taking the maximum in the sum over $I$.

\subsection{Bounds for Dirichlet polynomials}

We need several mean, large and pointwise value results on Dirichlet polynomials. The following lemma is one of the basic tools.

Lemma 3. (Mean value theorem for Dirichlet polynomials) Let $N \geq 1$ and $F(s)=$ $\sum_{n \sim N} \frac{a_{n}}{n^{s}}$, where $a_{n}$ are any complex numbers. Then

$$
\int_{-T}^{T}|F(i t)|^{2} d t \ll(N+T) \sum_{n \sim N}\left|a_{n}\right|^{2} .
$$

Proof. See for example Iwaniec and Kowalski's book [12, Chapter 9].

If the coefficients $a_{n}$ are supported on the primes or almost primes and are of size $\asymp \frac{1}{n}$, the sum $\sum_{n \sim N}\left|a_{n}\right|^{2}$ is essentially $\frac{1}{N \log N}$. However, in some places in the proofs of Theorems 1. 2 and 3 , it is vital to save one more logarithm in such a situation. This is enabled by an improved mean value theorem.

Lemma 4. (Improved mean value theorem) Let $N$ and $F(s)$ be as above. We have

$$
\int_{-T}^{T}|F(i t)|^{2} d t \ll T \sum_{n \sim N}\left|a_{n}\right|^{2}+T \sum_{1 \leq h \leq \frac{N}{T}} \sum_{\substack{m-n=h \\ m, n \sim N}}\left|a_{m}\right|\left|a_{n}\right| .
$$

Remark 5. The number of solutions to $m-n=h$, with $m$ and $n$ primes and $m, n \sim N$, is $\ll \frac{N^{2}}{\log ^{2} N} \cdot \frac{h}{\varphi(h)}$ (with $\varphi$ Euler's totient function), which follows easily from Brun's sieve, for example. If $T \leq \frac{N}{h}, h \geq \log N$ and $a_{n}$ is supported on the primes, the first sum in (6) turns out not to be problematic, so we indeed save essentially one additional logarithm with this lemma. We remark that if we have polynomials of length $N \leq T$, Lemma 4 reduces to the basic mean value theorem.

Proof. This follows from Lemma 7.1 in [12, Chapter 7], taking $Y=10 T$ there.

We also put into use a discrete mean value theorem, which is particularly useful when we take the mean square over a rather small set of points.

Lemma 5. (Halász-Montgomery inequality) Let $N$ and $F(s)$ be as before. Let $\mathcal{T} \subset[-T, T]$ be well-spaced, meaning that $t, u \in \mathcal{T}$ and $t \neq u$ imply $|t-u| \geq 1$. Then

$$
\sum_{t \in \mathcal{T}}|F(i t)|^{2} \ll\left(N+|\mathcal{T}| T^{\frac{1}{2}}\right)(\log T) \sum_{n \sim N}\left|a_{n}\right|^{2} .
$$


Proof. For a proof, see Iwaniec and Kowalski's book [12, Chapter 9].

In addition to mean value theorems, we need some large values theorems. We come across some very short Dirichlet polynomials, say of length $\ll T^{o(1)}$, and we make use of the fact that the coefficients of these polynomials are supported on the primes.

Lemma 6. Let $P \geq 1, V>0$ and

$$
F(s)=\sum_{p \sim P} \frac{a_{p}}{p^{s}}
$$

with $\left|a_{p}\right| \leq 1$. Let $\mathcal{T} \subset[-T, T]$ be a well-spaced set of points such that $|F(1+i t)| \geq V$ for each $t \in \mathcal{T}$. Then we have

$$
|\mathcal{T}| \ll T^{2 \frac{\log V^{-1}}{\log P}} V^{-2} \exp \left((1+o(1)) \frac{\log T}{\log P} \log \log T\right) .
$$

Remark 6. We may also apply this lemma to polynomials not supported on primes, provided that $P \gg X^{\varepsilon}$ for some $\varepsilon>0$. In this case, the lemma is essentially the mean value theorem applied to a suitable moment of the polynomial.

Proof. This is Lemma 8 in the paper [15]. There a factor of 2 occurs instead of $1+o(1)$ in the last exponential, but the exact same proof works with the factor $1+o(1)$.

For proving Theorem 5, we also need a large values theorem designed for long polynomials. The reason for presenting it along with the lemmas for Theorem 4 is that combining it with the other lemmas already gives the exponent $c=5+\varepsilon$ for $E_{2}$ numbers. The large values result is a theorem of Jutila that improves on the better known Huxley's large values theorem.

Lemma 7. (Jutila's large values theorem). Let $F(s)=\sum_{n \sim N} \frac{a_{n}}{n^{s}}$ with $\left|a_{n}\right| \leq d_{r}(n)$ for some fixed $r$. Let $\mathcal{T} \subset[-T, T]$ be a well-spaced set such that $|F(1+i t)| \geq V$ for $t \in \mathcal{T}$, and let $k$ be any positive integer. We have

$$
|\mathcal{T}| \ll\left(V^{-2}+\frac{T}{N^{2}} V^{-6+\frac{2}{k}}+V^{-8 k} \frac{T}{N^{2 k}}\right)(N T)^{o(1)} .
$$

Proof. The proof can be found in Jutila's paper [14]. We apply formula (1.4) there to $F(s)^{\ell}$, and have $G=\sum_{n \sim N} \frac{\left|a_{n}\right|^{2}}{n^{2}} \ll(N T)^{o(1)} N^{-1}$ in the notation of that paper.

In some cases in the proof of Theorem 4 , there will be polynomials supported on primes or almost primes for which the best we can do is apply a pointwise bound. These bounds follow in the end from Vinogradov's zero-free region.

Lemma 8. Let

$$
P(s)=\sum_{n_{1} \cdots n_{k} \sim N} g_{1}\left(n_{1}\right) \cdots g_{k}\left(n_{k}\right)\left(n_{1} \cdots n_{k}\right)^{-s}
$$


where $k \geq 1$ is a fixed integer and each $g_{i}$ is either the Möbius function, the characteristic function of the primes, the identity function, or the logarithm function. We have

$$
|P(1+i t)| \ll \exp \left(-(\log N)^{\frac{1}{10}}\right)
$$

when $\exp \left((\log N)^{\frac{1}{3}}\right) \leq|t| \leq N^{A \log \log N}$ for any fixed $A>0$.

Proof. For $k=1$, the claim follows directly from Perron's formula and Vinogradov's zerofree region, so let $k \geq 2$. We may assume that $n_{1}, \ldots, n_{k}$ belong to some dyadic intervals $I_{1}, \ldots, I_{k}$ such that $I_{k}=[a, b]$ with $a \gg N^{\frac{1}{k}}, b \ll N$. Now

$$
\begin{aligned}
& \sum_{n_{1} \in I_{1}, \ldots, n_{k-1} \in I_{k-1}} g\left(n_{1}\right) \cdots g\left(n_{k-1}\right)\left(n_{1} \cdots n_{k-1}\right)^{-1-i t} \sum_{\substack{n_{k} \in I_{k} \\
n_{k} \sim \bar{N}_{1} \cdots n_{k-1}}} g\left(n_{k}\right) n_{k}^{-1-i t} \\
& \ll(\log N)^{O(1)} \sum_{\substack{n_{1} \in I_{1}, \ldots, n_{k-1} \in I_{k-1}\\
}}\left(n_{1} \cdots n_{k-1}\right)^{-1} \cdot \exp \left(-\frac{\log N^{\frac{1}{k}}}{(\log t)^{\frac{2}{3}+\varepsilon}}\right) \\
& \ll \exp \left(-(\log N)^{\frac{1}{10}}\right),
\end{aligned}
$$

as wanted.

\subsection{Moments of Dirichlet polynomials}

We need Watt's result on the twisted fourth moment of zeta sums (see Subsection 1.2 for the definition of zeta sums). This bound comes into play when we estimate the mean square of a product of Dirichlet polynomials where one of the polynomials is a long zeta sum.

Lemma 9. (Watt). Let $T \geq T_{0} \geq T^{\varepsilon}, T^{1+o(1)} \gg M, N \geq 1$. Define the Dirichlet polynomials $N(s)=\sum_{n \sim N} n^{-s}$ or $N(s)=\sum_{n \sim N}(\log n) n^{-s}$ and $M(s)=\sum_{m \sim M} \frac{a_{m}}{m^{s}}$ with $a_{m}$ any complex numbers. We have

$$
\int_{T_{0}}^{T}|N(1+i t)|^{4}|M(1+i t)|^{2} d t \ll\left(\frac{T}{M N^{2}}\left(1+M^{2} T^{-\frac{1}{2}}\right)+\frac{1}{T_{0}^{3}}\right) T^{o(1)} \max _{m \sim M}\left|a_{m}\right|^{2} .
$$

Proof. An easy partial summation argument shows that we may assume $N(s)=\sum_{n \sim N} n^{-s}$. The lemma will be reduced to Watt's original twisted moment result [19], where $N(s)$ is replaced with $\zeta(s)$. It is well-known that $|N(1+i t)| \ll \frac{1}{t}$ for $N \geq t \geq 1$ (see [12, Chapter 8]), so

$$
\begin{aligned}
\int_{T_{0}}^{N}|N(1+i t)|^{4}|M(1+i t)|^{2} d t & \ll \max _{m \sim M}\left|a_{m}\right|^{2} \int_{T_{0}}^{T} \frac{1}{t^{4}} d t \cdot T^{o(1)} \\
& \ll \frac{T^{o(1)}}{T_{0}^{3}} \max _{m \sim M}\left|a_{m}\right|^{2} .
\end{aligned}
$$

Now it suffices to consider the integrals over dyadic intervals $[U, 2 U]$ with $N \leq U \leq T$. These are bounded as in Lemma 2 of [1] (using Watt's result and simple considerations), since translating the results there from the line $\Re(s)=\frac{1}{2}$ to the line $\Re(s)=1$ is an easy matter (and the bound in [1] should be multiplied by $\max _{m \sim M}\left|a_{m}\right|^{2}$, as we do not assume $\left.\left|a_{m}\right| \leq 1\right)$. 


\subsection{Sieve estimates}

There are occasions in the proofs of Theorems 4 and 5 where our Dirichlet polynomials are too long, and we need a device for splitting them into shorter ones. This is enabled by Heath-Brown's identity and the decomposition resulting from it, which tells that either our Dirichlet polynomial can be replaced with a product of many polynomials, which is desirable, or it can be replaced with products of zeta sums, in which case we can make use of Watt's theorem.

Definition 1. A Dirichlet polynomial $M(s)=\sum_{m \sim M} \frac{a_{m}}{m^{s}}$ with $\left|a_{n}\right| \ll d_{r}(n)$ for fixed $r$ is called prime-factored if, for each $A>0$, we have $|M(1+i t)| \ll_{A}(\log M)^{-A}$ for $\exp \left((\log M)^{\frac{1}{3}}\right) \leq t \leq M^{A \log \log M}$.

Lemma 10. (Heath-Brown's decomposition) Let an integer $k \geq 1$ and a real number $\delta>0$ be fixed, and let $T \geq 2$. Define $P(s)=\sum_{P \leq p<P^{\prime}} p^{-s}$ with $P \gg T^{\delta}, P^{\prime} \in\left[P+\frac{P}{\log T}, 2 P\right]$. There exist Dirichlet polynomials $G_{1}(s), \ldots, G_{L}(s)$ and a constant $C>0$ such that

$$
|P(1+i t)| \ll\left(\log ^{C} X\right)\left(\left|G_{1}(1+i t)\right|+\cdots+\left|G_{L}(1+i t)\right|\right) \quad \text { for all } \quad t \in[-T, T]
$$

with $L \leq \log ^{C} X$, each $G_{j}(s)$ being of the form

$$
G_{j}(s)=\prod_{i \leq J_{j}} M_{i}(s), \quad J_{j} \leq 2 k,
$$

with $M_{i}(s)$ prime-factored Dirichlet polynomials (which depend on $j$ ), whose lengths satisfy $M_{1} \cdots M_{J}=X^{1+o(1)}, M_{i} \gg \exp \left(\frac{\log P}{\log \log P}\right)$. Additionally, each $M_{i}(s)$ with $M_{i}>X^{\frac{1}{k}}$ is a zeta sum.

Proof. For a similar bound, see Harman's book [10, Chapter 7]. It suffices to prove an analogous result for the polynomial $\sum_{P \leq n<P^{\prime}} \Lambda(n) n^{-s}$ and use summation by parts. We take $f(n)=n^{-1-i t} 1_{\left[P, P^{\prime}\right]}(n)$ in the general Heath-Brown identity [11] for $\sum_{n \leq N} f(n) \Lambda(n)$, splitting each resulting variables into dyadic intervals, and separating the variables with Perron's formula. The summation condition in Heath-Brown's identity guarantees that of the arising polynomials only the zeta sums can have length $>X^{\frac{1}{k}}$. If there are any polynomials of length $\ll \exp \left(\frac{\log P}{\log \log P}\right)$, these can simply be estimated trivially. The fact that the remaining polynomials of length $\gg \exp \left(\frac{\log P}{\log \log P}\right)$ are prime-factored follows from the fact that they have as their coefficients one of the sequences $(1),(\log n)$ and $(\mu(n))$, so that Lemma 8 gives a pointwise saving of $\ll_{A}(\log P)^{-A}$.

There is one more lemma that we need on the coefficients of Dirichlet polynomials arising from almost primes. We need to bound the following quantities that are related to the quantities occurring in the improved mean value theorem for Dirichlet polynomials. 
Definition 2. For any sequence $\left(a_{n}\right)$ of complex numbers, set $X_{1}=\exp \left(\frac{\log X}{(\log \log X)^{4}}\right)$ and

$$
\begin{aligned}
& S_{1}\left(X,\left(a_{n}\right)\right)=\max _{\substack{X \\
X_{1} \leq Y \leq 4 X \\
1 \leq H \leq \log 10 X}} H \sum_{Y \leq n \leq Y+\frac{Y}{H}} \frac{\left|a_{n}\right|^{2}}{n}, \\
& S_{2}\left(X,\left(a_{n}\right)\right)=\max _{\substack{X \\
X_{1} \leq Y \leq 4 X \\
1 \leq H \leq \log ^{10} X}} H \sum_{1 \leq h \leq \frac{X}{T}} \sum_{Y \leq n \leq Y+\frac{Y}{H}} \frac{\left|a_{n}\right|\left|a_{n+h}\right|}{n} .
\end{aligned}
$$

We get bounds of size essentially $\frac{1}{\log X}$ and $\frac{X}{T \log ^{2} X}$ for $S_{1}\left(X,\left(a_{n}\right)\right)$ and $S_{2}\left(X,\left(a_{n}\right)\right)$, respectively, under the assumptions of the next lemma.

Lemma 11. Let $Z_{r} \geq \cdots \geq Z_{1} \geq 1$ for a fixed $r$ with $Z_{r} \geq \exp \left(\frac{\log X}{(\log \log X)^{3}}\right), Z_{r} \leq z \leq 4 X$, and

$$
\left.\mathcal{Q}=\left\{n \leq 4 X: n=p_{1} \cdots p_{r} m, p_{i} \in\left[Z_{i}, Z_{i}^{2}\right],(m, \mathcal{P}(z))=1\right)\right\} .
$$

Let $\left|a_{n}\right| \leq 1_{\mathcal{Q}}(n)$, and let $S_{1}\left(X,\left(a_{n}\right)\right)$ and $S_{2}\left(X,\left(a_{n}\right)\right)$ be as defined above. Then

$$
S_{1}\left(X,\left(a_{n}\right)\right) \ll \frac{1}{\log z} \quad \text { and } \quad S_{2}\left(X,\left(a_{n}\right)\right) \ll \frac{1}{\log ^{2} z} \cdot \frac{X}{T} .
$$

Remark 7. Notice that we could also take as the set $\mathcal{Q}$ the set

$$
\mathcal{Q}^{\prime}=\left\{n \leq 4 X: n=p_{1} \cdots p_{r} m, p_{i} \in\left[Z_{i}, Z_{i}^{2}\right],\left(m, \mathcal{P}\left(p_{r}\right)\right)=1\right\}
$$

or the set

$$
\mathcal{Q}^{\prime \prime}=\left\{n \leq 4 X: n=p_{1} \cdots p_{r}, p_{i} \in\left[Z_{i}, Z_{i}^{2}\right]\right\} .
$$

Indeed, the sizes of $\mathcal{Q}^{\prime}$ and $\mathcal{Q}^{\prime \prime}$ can be bounded by sizes of sets of the form given in the lemma (with the parameter $z=Z_{r}$ or $z=X^{\frac{1}{r-1}}$ ). This observation will be used subsequently.

Proof. Let $S(A, \mathbb{P}, z)$ count the numbers in $A$ having no prime factors below $z$, and let $\Pi$ be the product of all primes in $\bigcup_{i=1}^{r}\left[Z_{i}, Z_{i}^{2}\right] \cap[1, z]$. Brun's sieve yields

$$
\begin{aligned}
S_{1}\left(X,\left(a_{n}\right)\right) & \ll \max _{\substack{X \\
X_{1} \leq Y \leq 4 X \\
1 \leq H \leq \log ^{10} X}} \frac{H}{Y} \cdot\left|\left[Y, Y+\frac{Y}{H}\right] \cap \mathcal{Q}\right| \\
& \ll \max _{\substack{X \\
X_{1} \leq Y \leq 4 X \\
1 \leq H \leq \log ^{10} X}} \frac{H}{Y} \cdot\left|\left\{n \in\left[Y, Y+\frac{Y}{H}\right]:\left(n, \frac{\mathcal{P}(z)}{\Pi}\right)=1\right\}\right| \\
& \ll \max _{\substack{X \\
X_{1} \leq Y \leq 4 X}} \frac{H}{Y} \cdot\left(\frac{Y}{H \log z}+z^{\frac{1}{2}}\right) \\
& \ll \frac{1}{\log z},
\end{aligned}
$$


since $z^{\frac{1}{2}} \leq(4 X)^{\frac{1}{2}} \ll \frac{Y}{H \log ^{2} z}$.

Furthermore, Brun's sieve also yields

$$
\begin{aligned}
S_{2}\left(X,\left(a_{n}\right)\right) & \ll \max _{\substack{X_{1} \leq Y \leq 4 X \\
1 \leq H \leq \log 10}} \frac{H}{Y} \sum_{1 \leq h \leq \frac{X}{T}}\left|\left\{n \in\left[Y, Y+\frac{Y}{H}\right]:\left(n(n+h), \frac{\mathcal{P}(z)}{\Pi}\right)=1\right\}\right| \\
& \ll \max _{\substack{X_{1} \leq Y \leq 4 X \\
1 \leq H \leq \log X}} \frac{H}{Y} \cdot \sum_{1 \leq h \leq \frac{X}{T}} \frac{h}{\varphi(h)}\left(\frac{Y}{H \log ^{2} z}+z^{\frac{1}{2}}\right) \\
& \ll \frac{1}{\log ^{2} z} \cdot \frac{X}{T},
\end{aligned}
$$

by the elementary bound $\sum_{m \leq M} \frac{m}{\varphi(m)} \ll M$. This proves the statement.

\section{Mean squares of Dirichlet polynomials}

With all the necessary lemmas available, we are ready to present the propositions that quickly lead to Theorem 4 and are also necessary in proving Theorem 5.

Proposition 1. Let $X \geq 1, T \geq T_{0}=X^{0.01}, 0 \leq \alpha_{1} \leq 1$ and $1 \leq P \ll X^{o(1)}$, where $P$ is a function of $X$. Define

$$
K(s)=\sum_{n \sim \frac{X}{P}} \frac{a_{n}}{n^{s}} \quad \text { and } \quad P(s)=\sum_{p \sim P} \frac{b_{p}}{p^{s}},
$$

where $a_{n}$ and $b_{p}$ are arbitrary complex numbers. Denoting

$$
\mathcal{T}_{1}=\left\{t \in\left[T_{0}, T\right]:|P(1+i t)| \leq P^{-\alpha_{1}}\right\}
$$

we have

$$
\int_{\mathcal{T}_{1}}|K(1+i t) P(1+i t)|^{2} d t \ll \frac{T}{X} \cdot P^{1-2 \alpha_{1}}\left(S_{1}\left(\frac{X}{P},\left(a_{n}\right)\right)+S_{2}\left(\frac{X}{P},\left(a_{n}\right)\right)\right) .
$$

Proof. The improved mean value theorem (Lemma 4) and definition of $\mathcal{T}_{1}$ give

$$
\begin{aligned}
\int_{\mathcal{T}_{1}}|K(1+i t) P(1+i t)|^{2} d t & \ll P^{-2 \alpha_{1}} \int_{\mathcal{T}_{1}}|K(1+i t)|^{2} d t \\
& \ll P^{-2 \alpha_{1}}\left(T \sum_{k \sim \frac{X}{P}}\left|a_{k}\right|^{2}+T \sum_{\substack { 1 \leq h \leq \frac{X}{P T} \\
\begin{subarray}{c}{k, k^{\prime} \sim \\
k-k^{\prime}=h{ 1 \leq h \leq \frac { X } { P T } \\
\begin{subarray} { c } { k , k ^ { \prime } \sim \\
k - k ^ { \prime } = h } }\end{subarray}}\left|a_{k}\right|\left|a_{k^{\prime}}\right|\right) \\
& \ll P^{-2 \alpha_{1}}\left(\frac{T P}{X} S_{1}\left(\frac{X}{P},\left(a_{n}\right)\right)+\frac{T P}{X} S_{2}\left(\frac{X}{P},\left(a_{n}\right)\right)\right) \\
& =\frac{T}{X} \cdot P^{1-2 \alpha_{1}}\left(S_{1}\left(\frac{X}{P},\left(a_{n}\right)\right)+S_{2}\left(\frac{X}{P},\left(a_{n}\right)\right)\right),
\end{aligned}
$$

which was the claim. 
Proposition 2. Let $X \geq 1, T \geq T_{0}=X^{0.01}$ and $1 \leq P \ll X^{o(1)}$. Also let $0 \leq \alpha_{1}, \alpha_{2} \leq 1$ and let the Dirichlet polynomials $K(s)$ and $M(s)$ with $K=\frac{X}{M} \gg X^{\varepsilon}$ be

$$
K(s)=\sum_{n \sim K} \frac{a_{n}}{n^{s}} \quad \text { and } \quad M(s)=\sum_{m \sim M} \frac{c_{m}}{m^{s}},
$$

where $\left|c_{m}\right| \leq d_{r}(m)$ for fixed $r$, and $\left|a_{n}\right|=1_{S}(n)$ for some set $S$ whose elements have at most $r$ prime factors from $[P, 2 P]$ and have no prime factors in $\left[1, X^{0.01}\right] \backslash \bigcup_{i=1}^{r}\left[Z_{i}, Z_{i}^{2}\right]$ for some $Z_{i} \geq 1$. Write

$$
\begin{aligned}
P(s) & =\sum_{p \sim P} \frac{b_{p}}{p^{s}} \quad \text { with } \quad\left|b_{p}\right| \leq 1 \quad \text { and } \\
\mathcal{T} & =\left\{t \in\left[T_{0}, T\right]:|P(1+i t)| \geq P^{-\alpha_{1}} \text { and }|M(1+i t)| \leq M^{-\alpha_{2}}\right\} .
\end{aligned}
$$

We have

$$
\int_{\mathcal{T}}|K(1+i t) M(1+i t)|^{2} d t \ll M^{-2 \alpha_{2}} P^{(2+10 \varepsilon) \alpha_{1} \ell} \cdot(\ell !)^{1+o(1)} \cdot\left(\frac{T}{X} \cdot \frac{1}{\log X}+\frac{1}{\log ^{2} X}\right),
$$

where $\ell=\left\lceil\frac{\log \frac{X}{K}}{\log P}\right\rceil$.

Remark 8. For products of three primes, our variables are picked so that the bound given by this proposition saves $X^{\varepsilon}$ over the trivial bound. However, for products of $k \geq 4$ primes, our savings are much more modest, and the factor $\frac{T}{X} \cdot \frac{1}{\log X}+\frac{1}{\log ^{2} X}$ becomes necessary.

Proof. This result is inspired by Lemma 13 in [15]. Using the fact that $|M(1+i t)|^{2} \leq$ $M^{-2 \alpha_{2}}\left(P^{\alpha_{1}}|P(1+i t)|\right)^{2 \ell}$ for $t \in \mathcal{T}$ and splitting polynomials into shorter ones, we have

$$
\begin{aligned}
\int_{\mathcal{T}}|K(1+i t) M(1+i t)|^{2} d t & \ll M^{-2 \alpha_{2}} P^{2 \alpha_{1} \ell} \int_{\mathcal{T}}\left|K(1+i t) P(1+i t)^{\ell}\right|^{2} d t \\
& \ll M^{-2 \alpha_{2}} P^{2 \alpha_{1} \ell} \ell^{2} \int_{\mathcal{T}}|A(1+i t)|^{2} d t
\end{aligned}
$$

where

$$
A(s)=\sum_{n \sim Y} \frac{A_{n}}{n^{s}}
$$

for some $K P^{\ell} \leq Y \leq 2 K(2 P)^{\ell}$ (so $X \leq Y \leq 2^{\ell} P X$ ), the coefficients $A_{n}$ satisfying

$$
\left|A_{n}\right| \leq \sum_{\substack{n=p_{1} \cdots p_{\ell} m \\ p_{i} \sim P \\ m \sim K}}\left|a_{m}\right|
$$

By the improved mean value theorem (Lemma 4), we see that (7) is bounded by

$$
\ll M^{-2 \alpha_{2}} P^{2 \alpha_{1} \ell} \ell^{2}\left(T \sum_{n \sim Y}\left|\frac{A_{n}}{n}\right|^{2}+T \sum_{1 \leq h \leq \frac{Y}{T}} \sum_{m-n=h} \frac{\left|A_{m}\right|\left|A_{n}\right|}{m n}\right)
$$


Note that $A_{n} \neq 0$ implies that $n$ has at most $\ell+r$ prime factors from $[P, 2 P]$ and that $n$ is coprime to

$$
\Pi=\prod_{\substack{p \leq X^{0.01} \\ p \notin \bigcup_{i=1}^{r}\left[Z_{i}, Z_{i}^{2}\right] \cup[P, 2 P]}} p .
$$

Consequently, $\left|A_{n}\right| \leq(\ell+r)$ !, and so

$$
\begin{aligned}
\sum_{n \sim Y}\left|\frac{A_{n}}{n}\right|^{2} & \leq \frac{1}{Y} \cdot(\ell+r) ! \sum_{n \sim Y} \frac{\left|A_{n}\right|}{n} \\
& \ll \frac{1}{Y}(\ell !)^{1+o(1)} \sum_{m \sim K} \frac{\left|a_{m}\right|}{m} \sum_{p_{1}, \ldots, p_{\ell} \sim P} \frac{1}{p_{1} \cdots p_{\ell}} \\
& \ll(\ell !)^{1+o(1)} \cdot \frac{1}{Y} \sum_{\substack{m \sim K \\
(m, \Pi)=1}} \frac{\left|a_{m}\right|}{m} \\
& \ll(\ell !)^{1+o(1)} \cdot \frac{1}{X \log X},
\end{aligned}
$$

where the last step comes from Brun's sieve and the facts that $Y \geq X$ and $K \gg X^{\varepsilon}$.

To deal with the second sum arising from the improved mean value theorem, notice that by Brun's sieve the number of $n \leq y$ with $(n(k n+h), \Pi)=1$ is $\ll \frac{y}{\log ^{2} y} \frac{h k}{\varphi(h k)}$ with an absolute implied constant. Since $\varphi(a b) \geq \varphi(a) \varphi(b)$ and $\frac{k}{\varphi(k)} \leq 2^{\ell}$ when $k$ has $\ell$ prime factors, we have

$$
\begin{aligned}
& \sum_{1 \leq h \leq \frac{Y}{T}} \sum_{n \sim Y} \frac{\left|A_{n}\right|\left|A_{n+h}\right|}{n(n+h)} \\
& \leq \frac{1}{Y^{2}} \cdot(\ell+r) ! \sum_{1 \leq h \leq \frac{Y}{T}} \sum_{p_{1}, \ldots, p_{\ell} \sim P} \sum_{\substack{(m, \Pi)=1 \\
\left(p_{1} \cdots p_{\ell} m+h, \Pi\right)=1 \\
m \leq \frac{2 Y}{p_{1} \cdots p_{\ell}}}} 1 \\
& \ll \frac{1}{Y^{2}} \cdot(\ell !)^{1+o(1)} \sum_{1 \leq h \leq \frac{Y}{T}} \sum_{p_{1}, \ldots, p_{\ell} \sim P} \frac{Y}{p_{1} \cdots p_{\ell} \log ^{2} \frac{Y}{p_{1} \cdots p_{\ell}}} \frac{p_{1} \cdots p_{\ell} h}{\varphi\left(p_{1} \cdots p_{\ell} h\right)} \\
& \ll \frac{1}{Y \log ^{2} Y}(\ell !)^{1+o(1)} \sum_{1 \leq h \leq \frac{Y}{T}} \frac{h}{\varphi(h)} \sum_{p_{1}, \ldots, p_{\ell} \sim P} \frac{1}{p_{1} \cdots p_{\ell}} \\
& \ll \frac{1}{T}(\ell !)^{1+o(1)} \frac{1}{\log ^{2} X},
\end{aligned}
$$

as desired.

Proposition 3. Let $X^{1+o(1)} \geq T \geq T_{0}=X^{0.01}$ and $0 \leq \alpha_{1} \leq 1$. Furthermore, let

$$
P(s)=\sum_{p \sim P} \frac{a_{p}}{p^{s}}, \quad \text { and } \quad M(s)=\sum_{M \leq q \leq M^{\prime}} \frac{1}{q^{s}},
$$


with $\left|a_{p}\right| \leq 1, M^{\prime} \in\left[M+\frac{M}{\log P}, 2 M\right], \log X \leq P \ll X^{o(1)}$ and $P M=X^{1+o(1)}$, and let

$$
\mathcal{U}=\left\{t \in\left[T_{0}, T\right]:|P(1+i t)| \geq P^{-\alpha_{1}}\right\} .
$$

Then, for $\ell=\left\lfloor\varepsilon \frac{\log X}{\log P}\right\rfloor$,

$$
\begin{aligned}
& \int_{\mathcal{U}}|P(1+i t) M(1+i t)|^{2} d t \\
& \ll\left(P^{2 \alpha_{1}-1} \log ^{2} X\right)^{(1+o(1)) \ell} X^{o(1)}+(\log X)^{-100}\left(1+\frac{\left|\mathcal{U}^{\prime}\right| T^{\frac{1}{2}}}{X^{\frac{2}{3}-o(1)}}\right)
\end{aligned}
$$

for some well-spaced set $\mathcal{U}^{\prime} \subset \mathcal{U}$.

Proof. Heath-Brown's decomposition (Lemma 10 with $k=3$ allows us to write, for some $C>0$,

$$
|M(1+i t)| \ll\left(\log ^{C} X\right)\left(\left|G_{1}(1+i t)\right|+\cdots+\left|G_{L}(1+i t)\right|\right)
$$

with $L \leq \log ^{C} X$. Here each $G_{j}(s)$ is either of the form

$G_{j}(s)=M_{1}(s) M_{2}(s) M_{3}(s), M_{1} M_{2} M_{3}=X^{1+o(1)}, M_{1} \geq M_{2} \geq M_{3}, M_{3} \geq \exp \left(\frac{\log X}{2 \log \log X}\right)$

with $M_{i}(s)$ prime-factored polynomials, or of the form

$$
G_{j}(s)=N_{1}(s) N_{2}(s), N_{1} N_{2}=X^{1+o(1)}, N_{1} \geq N_{2}
$$

with $N_{i}(s)$ zeta sums (it is possible that $N_{2}(s)$ is the constant polynomial $1^{-s}$ ). It suffices to bound the contributions of the zeta sums and the prime-factored polynomials separately.

We look at the zeta sums first. We split the integration domain into dyadic intervals $\left[T_{1}, 2 T_{1}\right]$ with $T_{0} \leq T_{1} \leq T$. Keeping in mind that $N_{1} \geq X^{\frac{1}{2}-o(1)}, P^{\ell}=X^{\varepsilon+o(1)}$, and $\left|P(1+i t) P^{\alpha_{1}}\right|^{2 \ell} \geq 1$ for $t \in \mathcal{U}$, Cauchy-Schwarz and Watt's theorem (Lemma 9 yield

$$
\begin{aligned}
& \int_{\mathcal{U} \cap\left[T_{1}, 2 T_{1}\right]}\left|P(1+i t) N_{1}(1+i t) N_{2}(1+i t)\right|^{2} d t \\
& \ll P^{2 \alpha_{1} \ell} \int_{\mathcal{U} \cap\left[T_{1}, 2 T_{1}\right]}\left|N_{1}(1+i t) N_{2}(1+i t) P(1+i t)^{\ell}\right|^{2} d t \\
& \ll P^{2 \alpha_{1} \ell}\left(\int_{T_{1}}^{2 T}\left|N_{1}(1+i t)\right|^{4}|P(1+i t)|^{4 \ell} d t\right)^{\frac{1}{2}} \cdot\left(\int_{T_{1}}^{2 T_{1}}\left|N_{2}(1+i t)\right|^{4} d t\right)^{\frac{1}{2}} \\
& \ll P^{2 \alpha_{1} \ell} X^{o(1)}\left(\left(\frac{T_{1}+T_{1}^{\frac{1}{2}} P^{4 \ell}}{N_{1}^{2} P^{2 \ell}}+\frac{1}{T_{1}^{3}}\right)(2 \ell) !^{2}\right)^{\frac{1}{2}} \cdot\left(\frac{T_{1}+N_{2}^{2}}{N_{2}^{2}}\right)^{\frac{1}{2}} \\
& \ll P^{\left(2 \alpha_{1}-1\right) \ell} X^{o(1)} \cdot(\ell !)^{2+o(1)}+\frac{P^{2 \alpha_{1} \ell} X^{o(1)}(\ell !)^{2+o(1)}}{T_{0}} \\
& \ll\left(P^{2 \alpha_{1}-1} \log ^{2} X\right)^{(1+o(1)) \ell} X^{o(1)}+X^{-\varepsilon} .
\end{aligned}
$$


Combining the contributions of the dyadic intervals simply multiplies this bound by $\log X$.

To bound the contribution of the prime-factored polynomials, we first observe that

$$
\int_{\mathcal{U}}|P(1+i t) M(1+i t)|^{2} d t \ll \sum_{t \in \mathcal{U}^{\prime}}|P(1+i t) M(1+i t)|^{2}
$$

for some well-spaced $\mathcal{U}^{\prime} \subset \mathcal{U}$. We make use of the Halász-Montgomery inequality (Lemma 5), and of the prime-factored property applied to the polynomial $M_{3}$ with length $M_{3} \in$ $\left.\exp \left(\frac{\log X}{2 \log \log X}\right), X^{\frac{1}{3}+o(1)}\right]$, finding that

$$
\begin{aligned}
& \sum_{t \in \mathcal{U}^{\prime}}\left|P(1+i t) M_{1}(1+i t) M_{2}(1+i t) M_{3}(1+i t)\right|^{2} \\
& \ll(\log X)^{-100-D} \sum_{t \in \mathcal{U}^{\prime}}\left|P(1+i t) M_{1}(1+i t) M_{2}(1+i t)\right|^{2} \\
& \ll(\log X)^{-100-2 C}\left(1+\frac{T^{\frac{1}{2}}\left|\mathcal{U}^{\prime}\right|}{X^{\frac{2}{3}-o(1)}}\right),
\end{aligned}
$$

where $D$ is so large that $D-2 C-1$ exceeds the power of logarithm arising from the mean square of the coefficients of the divisor-bounded polynomial $P(s) M_{1}(s) M_{2}(s)$. Now the statement is proved.

\section{Proof of Theorem 4}

The following proposition yields Theorem 4 (and hence Theorems 1 and 2 immediately, in view of the remarks of Subsection 2.1

Proposition 4. Let $k \geq 3$ be a fixed integer, $\varepsilon>0$ be small enough and $T_{0}=X^{0.01}$, as before. Define

$$
F(s)=\sum_{\substack{p_{1} \cdots p_{k} \sim X \\ P_{i} \leq p_{i} \leq P_{i}^{1+\varepsilon} \\ i \leq k-1}}\left(p_{1} \cdots p_{k}\right)^{-s}
$$

where $P_{i}$ are as in Theorem 4. Then, for $T \geq T_{0}$, we have

$$
\int_{T_{0}}^{T}|F(1+i t)|^{2} d t \ll\left(\frac{T P_{1} \log X}{X}+1\right) \cdot \frac{1}{\left(\log ^{2} X\right)\left(\log _{k} X\right)^{3}} .
$$

Proof. We make use of the ideas introduced in the paper [15] by Matomäki and Radziwiłł. Trivially, we may assume $T \leq X^{1+o(1)}$. Let $H=\left(\log _{k} X\right)^{3}$,

$$
Q_{v, H}(s)=\sum_{e^{\frac{v}{H}} \leq p<e^{\frac{v+1}{H}}} p^{-s},
$$


and for each $j=1, \ldots, k$,

$$
F_{v, H, j}(s)=\sum_{\substack{p_{1} \cdots p_{j-1} p_{j+1} \cdots p_{k} \sim X e^{-\frac{v}{H}} \\ P_{i} \leq p_{i} \leq P_{i}^{1+\varepsilon}, i \neq j, i \leq k-1}}\left(p_{1} \cdots p_{j-1} p_{j+1} \cdots p_{k}\right)^{-s} .
$$

Define $\alpha_{1}, \ldots, \alpha_{k-1}$ by $\alpha_{j}=10 j \varepsilon$ for $j \leq k-2$, and $\alpha_{k-1}=\frac{1}{12}-\varepsilon$, with $\varepsilon$ so small that $\alpha_{k-2} \leq \frac{\sqrt{\varepsilon}}{10}$. We split the domain of integration as $\left[T_{0}, T\right]=\mathcal{T}_{1} \cup \mathcal{T}_{2} \cup \cdots \cup \mathcal{T}_{k-1} \cup \mathcal{T}$. We write $t \in \mathcal{T}_{1}$ if

$$
\left|Q_{v, H}(1+i t)\right| \leq e^{-\frac{\alpha_{1} v}{H}}
$$

for all $v \in I_{1}=\left[H \log P_{1},(1+\varepsilon) H \log P_{1}\right]$. We define recursively $t \in \mathcal{T}_{j}$ for $j=2, \ldots, k-1$ if $t \notin \bigcup_{j^{\prime} \leq j-1} \mathcal{T}_{j^{\prime}}$ but

$$
\left|Q_{v, H}(1+i t)\right| \leq e^{-\frac{\alpha_{j} v}{H}}
$$

for all $v \in I_{j}=\left[H \log P_{j},(1+\varepsilon) H \log P_{j}\right]$. Finally, we write

$$
\mathcal{T}=\left[T_{0}, T\right] \backslash \bigcup_{j=1}^{k-1} \mathcal{T}_{j}
$$

Lemma 2, with the notation of Subsection 2.5, yields

$$
\begin{aligned}
\int_{\mathcal{S}}|F(1+i t)|^{2} d t & \ll H^{2}\left(\log ^{2} P_{j}\right) \int_{\mathcal{S}}\left|Q_{v_{j}, H}(1+i t) F_{v_{j}, H, j}(1+i t)\right|^{2} d t \\
& +\frac{T}{H X}\left(S_{1}\left(X,\left(c_{n}\right)\right)+S_{2}\left(X,\left(c_{n}\right)\right)\right)
\end{aligned}
$$

for some $v_{j} \in I_{j}$, and any $\mathcal{S} \subset\left[T_{0}, T\right]$. The coefficients $c_{n}$ in the definitions of $S_{1}$ and $S_{2}$ are naturally the convolution of the absolute values of the coefficients of the polynomials $Q_{v_{j}, H}(s)$ and $F_{v_{j}, H, j}(s)$. By Lemma 11 and the remark related to it, the last two terms above contribute

$$
\begin{aligned}
& \ll \frac{T}{X} \cdot \frac{1}{H \log X}+\frac{1}{H \log ^{2} X} \\
& \ll\left(\frac{T P_{1} \log X}{X}+1\right) \cdot \frac{1}{H \log ^{2} X} .
\end{aligned}
$$

We choose $\mathcal{S}=\mathcal{T}_{1}, \ldots, \mathcal{T}_{k-1}, \mathcal{T}$ in (9). Summarizing, it suffices to estimate for each $j=$ $1, \ldots, k-1$ the quantity

$$
B_{j}:=H^{2}\left(\log ^{2} P_{j}\right) \int_{\mathcal{T}_{j}}\left|Q_{v_{j}, H}(1+i t) F_{v_{j}, H, j}(1+i t)\right|^{2} d t
$$

where $v_{j} \in\left[H \log P_{j},(1+\varepsilon) H \log P_{j}\right]$ is chosen so that the integral is maximal, and additionally the quantity

$$
B:=H^{2}\left(\log ^{2} X\right) \int_{\mathcal{T}}\left|Q_{v_{k}, H}(1+i t) F_{v_{k}, H, k}(1+i t)\right|^{2} d t,
$$


where $v_{k} \in\left[H \log \frac{X}{\left(P_{1} \cdots P_{k-1}\right)^{1+\varepsilon}}, H \log \frac{2 X}{P_{1} \cdots P_{k-1}}\right]$ is also picked so that the integral is maximized.

The integral over $\mathcal{T}_{1}$ is bounded with the help of Proposition 1. We take $K(s)=F_{v_{1}, H, 1}(s)$ and $P(s)=Q_{v_{1}, H}(s)$. Now Lemma 11 and Proposition 1 result in

$$
\begin{aligned}
B_{1} & \ll H^{2}\left(\log ^{2} P_{1}\right) P_{1}^{1+\varepsilon-2 \alpha_{1}} \frac{T}{X}\left(\frac{1}{\log X}+\frac{X}{P_{1} T} \cdot \frac{1}{\log ^{2} X}\right) \\
& \ll\left(\frac{T P_{1} \log X}{X}+1\right) \cdot \frac{P_{1}^{10 \varepsilon-2 \alpha_{1}}}{\log ^{2} X},
\end{aligned}
$$

and this is an admissible bound, since $\alpha_{1}=10 \varepsilon$ and $P_{1} \gg\left(\log _{k} X\right)^{\varepsilon^{-1}}$.

For the integral over $\mathcal{T}_{j}$ with $2 \leq j \leq k-1$ we use Proposition 2 , with $K(s)=F_{v_{j}, H, j}(s), M(s)=$ $Q_{v_{j}, H}(s)$ and $P(s)=Q_{v_{j-1}, H}(s)$, and for $\ell=\left\lceil\frac{\log P_{j}}{\log P_{j-1}}\right\rceil$ deduce

$$
\begin{aligned}
B_{j} \ll & H^{2}\left(\log ^{2} P_{j}\right) P_{j}^{-2 \alpha_{j}} \cdot P_{j-1}^{(2+10 \varepsilon) \alpha_{j-1} \ell} \\
& \cdot(\ell !)^{1+o(1)} \cdot\left(\frac{T}{X \log X}+\frac{1}{\log ^{2} X}\right) \\
& \ll P_{j-1}^{10} P_{j}^{2\left(\alpha_{j-1}-\alpha_{j}\right)+10 \varepsilon+(1+\varepsilon) \frac{\log \log P_{j}}{\log P_{j-1}}}\left(\frac{T P_{1} \log X}{X}+1\right) \frac{1}{\log ^{2} X} .
\end{aligned}
$$

For $2 \leq j \leq k-2$, we have $\frac{\log \log P_{j}}{\log P_{j-1}} \leq 2 \varepsilon$ and $\alpha_{j}-\alpha_{j-1}=10 \varepsilon$, so the definitions of $P_{j-1}$ and $P_{j}$ result in

$$
B_{j} \ll\left(\frac{T P_{1} \log X}{X}+1\right) \frac{1}{\log ^{2} X}\left(\log _{k} X\right)^{-3}
$$

as wanted. For $j=k-1$, we have $\alpha_{k-2} \leq \frac{\sqrt{\varepsilon}}{10}, \alpha_{k-1}=\frac{1}{12}-\varepsilon$ and $P_{k-1}=(\log X)^{\varepsilon^{-2}}$, so taking $j=k-1$ in the above computation gives

$$
B_{k-1} \ll P_{k-1}^{-\frac{1}{6}+\frac{1}{4} \sqrt{\varepsilon}+\frac{1+\varepsilon}{6+10 \sqrt{\varepsilon}}} \ll P_{k-1}^{-\varepsilon} \ll(\log X)^{-\varepsilon^{-1}},
$$

and therefore the case of $\mathcal{T}_{k-1}$ has been dealt with.

Finally, the integral over $\mathcal{T}$ is estimated using Proposition 3 with $P(s)=Q_{v_{k-1}, H}(s)$ and $M(s)=Q_{v_{k}, H}(s)$. Denoting $\ell=\left\lfloor\varepsilon \frac{\log X}{\log P_{k-1}}\right\rfloor$ and separating by Perron's formula the variable $p_{k-1}$ from the rest of the variables in $F_{v_{k}, H, k}(s)$ (and bounding the polynomial corresponding to the variables $p_{1}, \ldots, p_{k-2}$ by $\left.\ll 1\right)$, we see that

$$
\begin{aligned}
B & \ll H^{2}\left(\log ^{4} X\right) \int_{\mathcal{T}}\left|Q_{v_{k-1}, H}(1+i t) Q_{v_{k}, H}(1+i t)\right|^{2} d t \\
& \ll H^{2}\left(\log ^{4} X\right)\left(P_{k-1}^{-\frac{5}{6}+2 \varepsilon} \log ^{2} X\right)^{(1+o(1)) \ell} X^{o(1)}+(\log X)^{-95}\left(1+\frac{\left|\mathcal{T}^{\prime}\right| T^{\frac{1}{2}}}{X^{\frac{2}{3}-o(1)}}\right)
\end{aligned}
$$


for some well-spaced set $\mathcal{T}^{\prime} \subset \mathcal{T}$. Since $P_{k-1}=(\log X)^{\varepsilon^{-2}}$, the first term is $\ll X^{-\frac{\varepsilon}{3}}$. In addition, Lemma 6 allows us to bound the size of $\mathcal{T}^{\prime}$ by

$$
\left|\mathcal{T}^{\prime}\right| \ll T^{2 \alpha_{k-1}} P_{k-1}^{2} X^{\left(\varepsilon^{2}+o(1)\right)} \ll X^{\frac{1}{6}-\frac{\varepsilon}{2}},
$$

because $\alpha_{k-1}=\frac{1}{12}-\varepsilon$. Therefore, the integral over $\mathcal{T}$ is $\ll(\log X)^{-95}$. In conclusion, we deduced the bound

$$
B_{1}+\cdots+B_{k-1}+B \ll\left(\frac{T P_{1} \log X}{X}+1\right) \cdot \frac{1}{H \log ^{2} X},
$$

which finishes the proof of this proposition and of Theorem 4 .

\subsection{A corollary on products of two primes}

As a byproduct of the methods above, we arrive at the exponent $c=5+\varepsilon$ for products of two primes, which already replicates Mikawa's exponent for $P_{2}$ numbers ${ }^{2}$. Similarly as for products of three or more primes, it suffices to prove

$$
\int_{T_{0}}^{T}|F(1+i t)|^{2} d t=o\left(\left(\frac{T P_{1} \log X}{X}+1\right) \cdot \frac{1}{(\log X)^{2+\varepsilon}}\right),
$$

where

$$
F(s)=\sum_{\substack{p_{1} p_{2} \sim X \\ P_{1} \leq p_{1}<P_{1}^{1+\varepsilon}}}\left(p_{1} p_{2}\right)^{-s},
$$

and $P_{1}=\log ^{a} X$ with $a=4+\varepsilon$. We may again suppose $T \leq X^{1+o(1)}$.

We can redefine the set $\mathcal{T}_{1}$ in the proof of Proposition 4 with the new values $P_{1}=\log ^{a} X$, $H=(\log X)^{3 \varepsilon}$, keeping the value $\alpha_{1}=10 \varepsilon$, and we see again from Proposition 1 that the mean square of $F(1+i t)$ over $\mathcal{T}_{1}$ is suitably small. For applying Propositions 2 and 3. we need more polynomials than the two that correspond to the variables $p_{1}$ and $p_{2}$ in (2). Indeed, Heath-Brown's decomposition (Lemma 10) enables splitting the polynomial corresponding to $p_{2}$ as $(\log X)^{O(1)}$ sums of the form $\left|M_{1}(s) M_{2}(s)\right|+\left|N_{1}(s) N_{2}(s)\right|$, where $M_{1}(s)$ and $M_{2}(s)$ are prime-factored Dirichlet polynomials with $M_{1} M_{2}=X^{1+o(1)}$, $\exp \left(\frac{\log X}{2 \log \log X}\right) \ll M_{1} \ll X^{\frac{1}{3}+o(1)}$ and $N_{1}(s)$ and $N_{2}(s)$ zeta sums with $N_{1} N_{2}=X^{1+o(1)}$. The contribution of the zeta sums over the complement of $\mathcal{T}_{1}$ can be managed easily with Watt's theorem, similarly as in the proof of Proposition 3 .

To estimate the contribution of the prime-factored polynomials $M_{i}(s)$, we redefine the set $\mathcal{T}_{2}$ as $\left\{t \in\left[T_{0}, T\right]:\left|M_{1}(1+i t)\right| \leq M_{1}^{-\alpha_{2}}\right\} \backslash \mathcal{T}_{1}$, and Proposition 2 (with $P(s)$ corresponding to $p_{1}$ and $\left.K(s)=M_{1}(s) M_{2}(s)\right)$ produces a valid bound ${ }^{3}$ in the $\mathcal{T}_{2}$ case, as

\footnotetext{
${ }^{2}$ Adding to the argument a small refinement from Subsection 5.1, as well as Proposition 5 which is rather similar to Proposition 3 , would already give $c$ somewhat smaller than 5 .

${ }^{3}$ This bound for $a$ arises by inserting $P_{j-1}=\log ^{a} X$ and $P_{j}=X^{1+o(1)}$ into formula 10 .
} 
long as $a \geq \frac{1}{2\left(\alpha_{2}-\alpha_{1}\right)}+100 \varepsilon$. We take $\alpha_{2}=\frac{1}{8}-\varepsilon$, which turns out to be the best choice here.

Finally, when considering the integral over the complement of $\mathcal{T}_{1} \cup \mathcal{T}_{2}$, instead of Proposition 3 we apply the simple inequality

$$
\int_{\mathcal{T}}\left|M_{1}(1+i t) M_{2}(1+i t)\right|^{2} d t \ll(\log X)^{-100}\left(1+\frac{\left|\mathcal{T}^{\prime}\right| T^{\frac{1}{2}}}{M_{2}}\right)
$$

for some well-spaced $\mathcal{T}^{\prime} \subset \mathcal{T}$, with $\mathcal{T} \subset\left[T_{0}, T\right]$ arbitrary. This inequality follows just from the prime-factored property of $M_{1}(s)$ combined with the Halász-Montgomery inequality (Lemma 5). Now, denoting $M_{1}=X^{\nu+o(1)}$, we need to have $\left|\mathcal{T}^{\prime}\right| \ll X^{\frac{1}{2}-\nu-\varepsilon^{2}}$ whenever

$$
\mathcal{T}^{\prime} \subset\left\{t \in\left[T_{0}, T\right]:\left|M_{1}(1+i t)\right| \geq M_{1}^{-\alpha_{2}}\right\}
$$

is well spaced. Jutila's large values theorem (Lemma 7) applied with $F(s)=M_{1}(s)^{\ell}$, $V=M_{1}^{-\left(\frac{1}{8}-\varepsilon\right) \ell}$ and $k=2, \ell \in\{2,3\}$ tells that

$$
\left|\mathcal{T}^{\prime}\right| \ll\left\{\begin{array}{l}
X^{\max \left\{\frac{\nu}{2},-\frac{11}{4} \nu+1,1-4 \nu\right\}-2 \varepsilon^{2}} \\
X^{\max \left\{\frac{3}{4} \nu,-\frac{33}{8} \nu+1,1-6 \nu\right\}-2 \varepsilon^{2}}
\end{array}\right.
$$

We know that $\nu \leq \frac{1}{3}+o(1)$, and for $\frac{2}{7} \leq \nu \leq \frac{1}{3}$ the first bound is $\ll X^{\frac{1}{2}-\nu-\varepsilon^{2}}$, while for $\frac{4}{25} \leq \nu \leq \frac{2}{7}$ the second bound is small enough.

In the case $\nu \leq \frac{4}{25}$, we may simply appeal to Lemma 6 to bound $\left|\mathcal{T}^{\prime}\right|$ (with $V=M_{1}^{-\alpha_{2}}$ ), and get

$$
\left|\mathcal{T}^{\prime}\right| \ll T^{2 \alpha_{2}} X^{2 \nu \alpha_{2}+o(1)} \ll X^{0.29+100 \varepsilon} \ll X^{\frac{1}{2}-\nu-\varepsilon}
$$

for $\alpha_{2}=\frac{1}{8}-\varepsilon$. This proves that $\alpha_{2}=\frac{1}{8}-\varepsilon$ was permissible, leading to $a=\frac{1}{2 \alpha_{2}}+C_{1} \varepsilon$, so the admissible exponent becomes $c=a+1 \leq 5+2 C_{1} \varepsilon$ (and $\varepsilon>0$ was arbitrary). The rest of the paper therefore deals with improving the value $c=5+\varepsilon$ to $c=3.51$, which will require several further ideas, along with the ones already introduced.

\section{Lemmas For TheORem 5}

\subsection{Exponent pairs}

In the proof of Theorem 5, several zeta sums arise, and in some instances it is useful to have a smallish, pointwise power saving in these sums. This is given by the theory of exponent pairs. We could compute a long list of exponent pairs and choose the optimal estimate depending on the length of the zeta sum, but it turns out that using a single suitable exponent pair improves the exponent $c$ for $E_{2}$ numbers by approximately 0.02 , while having more of them would have very little additional advantage, and would complicate the calculations. Therefore, instead of formulating the general definition of exponent pairs (found in [17, Chapter 3]), we write down the estimate coming from this specific pair. 
Lemma 12. Let

$$
\sigma(\nu)=-\min \left\{\frac{1-\nu}{126}-\frac{\nu}{21}, 0\right\}
$$

Then we have

$$
\sum_{n \in I} n^{-1-i t} \ll t^{-\sigma(\nu)+o(1)}
$$

for each $I=\left[N_{1}, N_{2}\right]$ with $t^{\nu} \leq N_{1} \leq N_{2} \ll t^{\nu+o(1)}$.

Proof. This follows immediately from the fact that $\left(\frac{1}{126}, \frac{20}{21}\right)$ is an exponent pair. For the proof of this, see Montgomery's book [17, Chapter 3].

\subsection{Lemmas on sieve weights}

For finding products of two primes on short intervals, we need some lemmas concerning sieve weights. In the cases of sums $\Sigma_{1}(h)$ and $\Sigma_{2}(h)$ in Subsection 6.2, there will be too few variables for finding cancellation in the mean square of the corresponding Dirichlet polynomials. However, introducing sieve weights to these sums, we get an additional variable which is summed over all integers in a certain range, and separating that variable gives a long zeta sum (because there are few variables), and Watt's theorem can be applied to this sum. Also in the case of these sums, we need to make use of an additional saving of a logarithm in the mean value theorem. However, here the coefficients are not supported on almost primes but are closely related to the Dirichlet convolution $\lambda_{n} * 1$, where $\lambda_{n}$ are the sieve weights. The sieve weights $\lambda_{n}$ can be taken to be those of Brun's pure sieve. Specifically, we take

$$
\lambda_{d}^{+}=\left\{\begin{array}{ll}
\mu(d), & \nu(d) \leq R, d \mid \mathcal{P}(w) \\
0 & \text { otherwise }
\end{array} \quad \lambda_{d}^{-}= \begin{cases}\mu(d), & \nu(d) \leq R+1, d \mid \mathcal{P}(w) \\
0 & \text { otherwise }\end{cases}\right.
$$

where the notations are as in Subsection 1.2 , and

$$
w=\exp \left(\frac{\log X}{(\log \log X)^{3}}\right) \quad \text { and } \quad R=2\left\lfloor(\log \log X)^{\frac{3}{2}}\right\rfloor .
$$

Since the support of $\lambda_{n} * 1$ contains in addition to almost primes only numbers having exceptionally many prime factors, we are able to save one logarithm factor in the mean values. This is done in the following lemma.

Lemma 13. Let $\lambda_{d}^{+}$and $\lambda_{d}^{-}$be the sieve weights of Brun's pure sieve with the above notations. Let $k \geq 0$ be a fixed integer, $R_{1}, \ldots, R_{k} \geq 1$ and

$$
a_{n}=\sum_{\substack{p_{1} \cdots p_{k} \mid n \\ R_{i} \leq p_{i} \leq R_{i}^{1+\varepsilon}}}\left|\sum_{n=p_{1} \cdots p_{k} d m} \lambda_{d}^{ \pm}\right|
$$


where either the sign + or - is chosen throughout (for $k=0$, we define $p_{1} \cdots p_{k}=1$ ). Then for $y \gg_{A} \frac{x}{\log ^{A} x}$ and $x \sim X$ we have

$$
\begin{aligned}
& \sum_{x \leq n \leq x+y}\left|a_{n}\right|^{2} \ll_{A}(\log \log X)^{O_{k}(1)} \frac{y}{\log X} \\
& \sum_{1 \leq h \leq \frac{x}{T}} \sum_{\substack{m-n=h \\
m, n \in[x, x+y]}}\left|a_{m}\right|\left|a_{n}\right| \ll_{A}(\log \log X)^{O_{k}(1)} \frac{X}{T} \cdot \frac{y^{2}}{\log ^{2} X} .
\end{aligned}
$$

For the proof of this lemma, we need a couple of other lemmas.

Lemma 14. For $x \geq 2$ and positive integer $\ell$, let

$$
\pi_{\ell}(x)=|\{n \in[1, x]: \nu(n)=\ell\}| .
$$

There exist absolute constants $K$ and $C$ such that

$$
\pi_{\ell}(x)<\frac{K x}{\log x} \frac{(\log \log x+C)^{\ell-1}}{(\ell-1) !}
$$

for all $\ell$ and $x \geq 2$.

Proof. This is an elementary result of Hardy and Ramanujan from [8].

Lemma 15. Let $a \geq 1$ be fixed, and let $R=2\left\lfloor(\log \log X)^{\frac{3}{2}}\right\rfloor$ as before. Then for any $A>0$

$$
\sum_{\substack{n \sim X \\ \nu(n) \geq R}} a^{\nu(n)} \ll_{a, A} \frac{X}{\log ^{A} X} .
$$

Proof. The sum in question can be written as

$$
\sum_{\ell \geq R} a^{\ell}|\{n \sim X: \nu(n)=\ell\}|
$$

and by Lemma 14 this is

$$
\begin{aligned}
& \ll \frac{X}{\log X} \sum_{\ell \geq R}\left(\frac{a e(\log \log X+C)}{\ell-1}\right)^{\ell-1} \\
& \ll_{a} X \cdot 2^{-R} \ll_{A} \frac{X}{\log ^{A} X}
\end{aligned}
$$

by the definition of $R$.

We can now proceed to proving Lemma 13 . 
Proof of Lemma 13. It suffices to consider the lower bound sieve weights. We assume $k \geq 1$, as the case $k=0$ is similar but a little simpler. Define $\theta_{n}=1 * \lambda_{n}^{-}$. We have

$$
\begin{aligned}
\theta_{n} & =\sum_{\substack{d|n \\
\nu(d) \leq R \\
d| \mathcal{P}(w)}} \mu(d) \\
& =\sum_{d \mid(n, \mathcal{P}(w))} \mu(d)+O\left(\sum_{\substack{d \mid n \\
\nu(d)>R}}|\mu(d)|\right) \\
& =1_{(n, \mathcal{P}(w))=1}+O\left(2^{\nu(n)} 1_{\nu(n)>R}\right) .
\end{aligned}
$$

Using this, we bound the sum (11). Denoting by $\Pi$ the product of all the primes in $\bigcup_{i=1}^{k}\left[R_{i}, R_{i}^{1+\varepsilon}\right] \cap[1, w]$, we observe that

$$
a_{n}=\sum_{\substack{p_{1} \cdots p_{k} \mid n \\ R_{i} \leq p_{i} \leq R_{i}^{1+\varepsilon}}}\left|\theta_{\frac{n}{p_{1} \cdots p_{k}}}\right| \leq \nu(n)^{k}\left(1_{\left(n, \frac{\mathcal{P}(w)}{\Pi}\right)=1}+2^{\nu(n)} 1_{\nu(n)>R}\right) .
$$

The contribution of the first term on the right-hand side of $(13)$ to the sum (11) is

$$
\ll \sum_{\substack{x \leq n \leq x+y \\\left(n, \frac{\mathcal{P}(w)}{\Pi}\right)=1}} \nu(n)^{2 k} \ll(\log \log X)^{O_{k}(1)} \sum_{\substack{x \leq n \leq x+y \\\left(n, \frac{\mathcal{P}(w)}{\Pi}\right)=1}} 1 \ll(\log \log X)^{O_{k}(1)} \frac{y}{\log X}
$$

by Brun's sieve and the fact that $\nu(n) \ll(\log \log X)^{3}$ when $(n, \mathcal{P}(w))=1$. On the other hand, the the second term on the right-hand side of $(13)$ contributes to (11) at most

$$
\ll \sum_{\substack{x \leq n \leq x+y \\ \nu(n) \geq R}} \nu(n)^{2 k} 4^{\nu(n)} \ll_{k} \sum_{\substack{x \leq n \leq x+y \\ \nu(n) \geq R}} 5^{\nu(n)} \ll_{A, k} \frac{X}{\log ^{A} X}
$$

by Lemma 15. This proves the first bound in Lemma 13

The second bound in Lemma 13 is proved analogously. The two terms in 13 can be combined in four ways into products of two terms (two of these are symmetric). One of the cases contributes to 12 at most

$$
\ll \sum_{1 \leq h \leq \frac{x}{T}} \sum_{\substack{m, n=h \\ m, n \in[x, x+y]}} \nu(m)^{k} \nu(n)^{k} 1_{\left(m, \frac{\mathcal{P}(w)}{\Pi}\right)=1} 1_{\left(n, \frac{\mathcal{P}(w)}{\Pi}\right)=1} \ll(\log \log X)^{O_{k}(1)} \frac{X}{T} \cdot \frac{y^{2}}{\log ^{2} X}
$$

by Brun's sieve. The two symmetric terms obtained by multiplying terms in 13 have an impact of

$$
\ll \sum_{1 \leq h \leq \frac{x}{T}} \sum_{\substack{m-n=h \\ m, n \in[x, x+y]}} \nu(m)^{k} \nu(n)^{k} 1_{\left(m, \frac{\mathcal{P}(w)}{\Pi}\right)=1} 2^{\nu(n)} 1_{\nu(n)>R}
$$

where the coefficients depending on $m$ can be bounded trivially, while the coefficients depending on $n$ save an arbitrary power of logarithm, as in formula (14). Finally, the fourth term arising from multiplication of $(13)$ also saves an arbitrary power of logarithm by the same argument. 


\section{Proof of Theorem 5}

Before proving Theorem 5, we need some preparation. Define

$$
S_{h}(x)=\sum_{\substack{x \leq p_{1} p \leq x+h \\ P_{1} \leq p_{1} \leq P_{1}^{1+\varepsilon}}} 1, \quad S_{X}=S_{X}(X),
$$

and set

$$
w=\exp \left(\frac{\log X}{(\log \log X)^{3}}\right) .
$$

We use Buchstab's identity twice to decompose

$$
\begin{aligned}
& S_{h}(x)=\sum_{\substack{x \leq p_{1} n \leq x+h \\
P_{1} \leq p_{1} \leq P_{1}^{1+\varepsilon} \\
(n, \mathcal{P}(w))=1 \\
n>1}} 1-\sum_{\substack{x \leq p_{1} q_{1} n \leq x+h \\
P_{1} \leq p_{1} \leq P_{1}^{1+\varepsilon} \\
w \leq q_{1}<\sqrt{x} \\
\left(n, \mathcal{P}\left(q_{1}\right)\right)=1 \\
n>1}} 1 \\
& =\sum_{\substack{x \leq p_{1} n \leq x+h \\
P_{1} \leq p_{1} \leq P_{1}^{1+\varepsilon} \\
(n, \mathcal{P}(w))=1 \\
n>1}} 1-\sum_{\substack{x \leq p_{1} q_{1} n \leq x+h \\
P_{1} \leq p_{1} \leq P_{1}^{1+\varepsilon} \\
w \leq q_{1}<\sqrt{x} \\
(n, \mathcal{P}(w))=1}} 1+\sum_{\substack{x \leq p_{1} q_{1} q_{2} n \leq x+h \\
P_{1} \leq p_{1} \leq P_{1}^{1+\varepsilon} \\
w \leq q_{2}<q_{1}<\sqrt{x} \\
\left(n, \mathcal{P}\left(q_{1}\right)\right)=1 \\
n>1}} 1 .
\end{aligned}
$$

Call these sums $\Sigma_{1}(h), \Sigma_{2}(h)$ and $\Sigma_{3}(h)$, respectively, and call the corresponding dyadic sums $\Sigma_{1}(X), \Sigma_{2}(X)$ and $\Sigma_{3}(X)$, respectively. We will divide $\Sigma_{3}(h)$ into two parts $\Sigma_{3}^{\prime}(h)$ and $\Sigma_{3}^{\prime \prime}(h)$ in such a way that $\Sigma_{1}(h), \Sigma_{2}(h)$ and $\Sigma_{3}^{\prime}(h)$ can be evaluated asymptotically, while the error from $\Sigma_{3}^{\prime \prime}(h)$ is manageable. To be precise, we will prove that

$$
\begin{aligned}
\frac{1}{h} S_{h}(x) & =\frac{1}{h}\left(\Sigma_{1}(h)-\Sigma_{2}(h)+\Sigma_{3}^{\prime}(h)+\Sigma_{3}^{\prime \prime}(h)\right) \\
& =\frac{1}{X}\left(\Sigma_{1}(X)-\Sigma_{2}(X)+\Sigma_{3}^{\prime}(X)\right)+\frac{1}{h} \Sigma_{3}^{\prime \prime}(h)+o\left(\frac{1}{\log X}\right) \\
& =\frac{1}{X} S_{X}+\frac{1}{h} \Sigma_{3}^{\prime \prime}(h)-\frac{1}{X} \Sigma_{3}^{\prime \prime}(X)+o\left(\frac{1}{\log X}\right) \\
& \geq \frac{1}{X} S_{X}-\frac{1}{X} \Sigma_{3}^{\prime \prime}(X)+o\left(\frac{1}{\log X}\right) \\
& \geq \varepsilon \cdot \frac{1}{X} S_{X}
\end{aligned}
$$

almost always, with the steps 15$)$ and $(16)$ being the nontrivial ones. This estimate will then immediately lead to Theorem 5 . To prove these statements, we require some auxiliary results for the cases of $\Sigma_{1}(h), \Sigma_{2}(h)$ and $\Sigma_{3}(h)$.

\subsection{Mean square bounds related to Theorem 5}

We need three additional mean square bounds to deal with the sums $\Sigma_{1}(h), \Sigma_{2}(h)$ and $\Sigma_{3}(h)$. The first is a relative of Proposition 3 and would already improve slightly the 
exponent $c=5+\varepsilon$ obtained from the proof of Theorem 4. It will not be applied directly in the proof of Theorem 5 , but instead as an ingredient in the proof of Proposition 7 .

Proposition 5. Let $X^{1+o(1)} \geq T \geq T_{0}=X^{0.01}$, and $0 \leq \alpha_{1} \leq 1$. Furthermore, let

$$
P(s)=\sum_{P \leq p \leq P^{\prime}} \frac{1}{p^{s}}, \quad M(s)=\sum_{m \sim M} \frac{b_{m}}{m^{s}},
$$

with $P=X^{\nu+o(1)}, P^{\prime} \in\left[P+\frac{P}{\log X}, 2 P\right], 0<\nu \leq \frac{1}{2},\left|b_{m}\right| \leq d_{r}(m)$ for fixed $r$, and $P M=X^{1+o(1)}$. Also let

$$
\mathcal{U}=\left\{t \in\left[T_{0}, T\right]:|P(1+i t)| \geq P^{-\alpha_{1}}\right\} .
$$

Then,

$$
\int_{\mathcal{U}}|P(1+i t) M(1+i t)|^{2} d t \ll(\log X)^{-100}+X^{\frac{1}{2}-\min \left\{2 \sigma(\nu), \frac{\nu}{2}\right\}+o(1)} \cdot \frac{\left|\mathcal{U}^{\prime}\right| P}{X}
$$

for some well-spaced $\mathcal{U}^{\prime} \subset \mathcal{U}$.

Proof. Note that Heath-Brown's decomposition (Lemma 10 gives

$$
|P(1+i t)| \ll\left(\log ^{C} X\right)\left(\left|G_{1}(1+i t)\right|+\cdots+\left|G_{L}(1+i t)\right|\right)
$$

with $L \leq \log ^{C} X$ and each $G_{j}(s)$ either of the form $G_{j}(s)=N(s)$ with $N(s)$ a zeta sum of length $P^{1-o(1)}$, or $G_{j}(s)=M_{1}(s) M_{2}(s)$ with $M_{1}$ and $M_{2}$ prime-factored polynomials of length $M_{1} \geq M_{2} \geq \exp \left(\frac{\log X}{\log \log X}\right), M_{1} M_{2}=P^{1-o(1)}$. To bound the contribution of the zeta sum, we divide the integral over $\mathcal{U}$ into integrals over dyadic intervals $\left[T_{1}, 2 T_{1}\right]$ with $T_{1} \in\left[T_{0}, T\right]$, and write $N=T_{1}^{\mu+o(1)}$ with $\mu \geq \nu$. If $\mu>1$, we know that $|N(1+i t)| \ll \frac{\log t}{t}$ and $M(1+i t) \ll(\log X)^{O(1)}$, so

$$
\int_{\mathcal{U} \cap\left[T_{1}, 2 T_{1}\right]}|M(1+i t) N(1+i t)|^{2} d t \ll \frac{(\log X)^{O(1)}}{T_{0}} .
$$

If $\mu \leq 1$, we first pick a well-spaced $\mathcal{U}^{\prime} \subset \mathcal{U}$ such that

$$
\int_{\mathcal{U}}|M(1+i t) N(1+i t)|^{2} d t \ll \sum_{t \in \mathcal{U}^{\prime}}|M(1+i t) N(1+i t)|^{2} .
$$

Now the Halász-Montgomery inequality and the the fact that $N(s)$ is a zeta sum give

$$
\begin{aligned}
\sum_{t \in \mathcal{U}^{\prime} \cap\left[T_{1}, 2 T_{1}\right]}|M(1+i t) N(1+i t)|^{2} & \ll T^{-2 \sigma(\nu)+o(1)} \sum_{t \in \mathcal{U}^{\prime} \cap\left[T_{1}, 2 T_{1}\right]}|M(1+i t)|^{2} \\
& \ll T^{-2 \sigma(\nu)+o(1)}\left(1+\frac{\left|\mathcal{U}^{\prime}\right| T_{1}^{\frac{1}{2}+o(1)}}{\frac{X}{P}}\right) .
\end{aligned}
$$


To deal with the contribution of the prime-factored polynomials $M_{i}(s)$, we may use the Halász-Montgomery inequality in a manner analogous to the above to obtain the estimate

$$
\int_{\mathcal{U}}\left|M(1+i t) M_{1}(1+i t) M_{2}(1+i t)\right|^{2} d t \ll(\log X)^{-100}\left(1+\frac{\left|\mathcal{U}^{\prime}\right| T^{\frac{1}{2}+o(1)}}{\frac{X}{P^{\frac{1}{2}}}}\right),
$$

since $M M_{1} \gg \frac{X^{1+o(1)}}{P^{\frac{1}{2}}}$ Taking the maximum of these two results produces the claimed bound.

Our second mean square bound is a type I estimate where we exploit a long zeta sum with the help of Watt's theorem. In the cases of $\Sigma_{1}(h)$ and $\Sigma_{2}(h)$, this is necessary, and in the case $\Sigma_{3}(h)$ it improves our exponent for Theorem 5 . A closely related estimate can be found for example in [10, Chapter 9].

Proposition 6. Let $X^{1+o(1)} \gg T \geq T_{0}$, and let $M(s), N(s), P(s)$ be Dirichlet polynomials with coefficients bounded by $X^{o(1)}$ and supported on the intervals $[M, 2 M],[N, 2 N],[P, 2 P]$, respectively. Denote $Q(s)=\sum_{m \sim Q} \frac{a_{m}}{m^{s}}$, and let $N(s)$ be a zeta sum. Suppose in addition that

$$
M N P=X^{1+o(1)}, P Q^{2} \leq X^{\frac{1}{4}}, M^{2} P \ll X^{1+o(1)} .
$$

Then

$$
\int_{T_{0}}^{T}|M(1+i t) N(1+i t) P(1+i t) Q(1+i t)|^{2} d t \ll X^{o(1)}\left(Q^{-1}+\frac{1}{T_{0}}\right) \max _{m \sim Q}\left|a_{m}\right|^{2} .
$$

Remark 9. In all our applications, the polynomial $Q(s)$ has length essentially $X^{\varepsilon}$, and it is used to win by $X^{\varepsilon^{2}}$, say, in our estimates.

Proof. We will reduce the proposition to Watt's theorem (Lemma 9). Divide the integration domain into dyadic intervals $\left[T_{1}, 2 T_{1}\right]$. By Cauchy-Schwarz, the mean value theorem and Watt's theorem, we see that

$$
\begin{aligned}
& \int_{T_{1}}^{2 T 1}|M(1+i t) N(1+i t) P(1+i t) Q(1+i t)|^{2} d t \\
& \ll\left(\int_{T_{1}}^{2 T 1}|N(1+i t)|^{4}\left|P(1+i t) Q(1+i t)^{2}\right|^{2} d t\right)^{\frac{1}{2}} \\
& \quad \cdot\left(\int_{T_{1}}^{2 T_{1}}|M(1+i t)|^{4}|P(1+i t)|^{2} d t\right)^{\frac{1}{2}} \\
& \ll\left(\left(\frac{T_{1}^{o(1)}\left(T_{1}+T_{1}^{\frac{1}{2}} P^{2} Q^{4}\right)}{N^{2} P Q^{2}}+\frac{T^{o(1)}}{T_{1}^{3}}\right) \max _{m \sim Q}\left|a_{m}\right|^{4}\right)^{\frac{1}{2}}\left(\frac{T_{1}+M^{2} P}{M^{2} P}\right)^{\frac{1}{2}} \\
& \ll\left(\left(\frac{T_{1}^{o(1)}\left(T_{1}+T_{1}^{\frac{1}{2}} P^{2} Q^{4}\right)}{N^{2} P Q^{2}}\right) \max _{m \sim Q}\left|a_{m}\right|^{4}\right)^{\frac{1}{2}}\left(\frac{T_{1}+M^{2} P}{M^{2} P}\right)^{\frac{1}{2}}+\frac{X^{o(1)}}{T_{0}} \max _{m \sim Q}\left|a_{m}\right|^{2} .
\end{aligned}
$$


Hence, we need

$$
\left(X+X^{\frac{1}{2}} P^{2} Q^{4}\right)\left(X+M^{2} P\right) \ll\left(M N P X^{o(1)}\right)^{2},
$$

and this is guaranteed by our conditions.

For the $\Sigma_{3}(h)$ case in Subsection 6.3, we also need the following mean square bound, which is somewhat analogous to Proposition 4 and is based on Propositions 1, 2 and 5 , but it will be clear only later how it is crucial for proving Theorem 5 .

Proposition 7. Let $0 \leq \nu \leq \frac{1}{2}, 0<\alpha_{2} \leq 1, a=\frac{1}{2 \alpha_{2}}+C_{2} \varepsilon, P_{1}=\log ^{a} X, X^{1+o(1)} \gg T \geq$ $T_{0}=X^{0.01}$, and $w \leq P_{2}=X^{\nu+o(1)}$ with $w=\exp \left(\frac{\log X}{(\log \log X)^{3}}\right)$. Also let

$$
G(s)=\sum_{\substack{p_{1} p_{2} p_{3} n \sim X \\ P_{i} \leq p_{i} \leq P_{i}^{1+\varepsilon} \\ p_{2}<p_{3} \\\left(n, \mathcal{P}\left(p_{2}\right)\right)=1 \\ n>1}} a_{n}\left(p_{1} p_{2} p_{3} n\right)^{-s},
$$

where $\left|a_{n}\right| \ll(\log X)^{\varepsilon}$. Suppose that for every Dirichlet polynomial $M(s)=\sum_{m \sim M} \frac{b_{m}}{m^{s}}$ with $\left|b_{m}\right| \leq d_{r}(m)$ for fixed $r$ and $M=X^{\nu+o(1)}$ any well-spaced set

$$
\mathcal{U}^{\prime} \subset\left\{t \in[0, T]:|M(1+i t)| \geq M^{-\alpha_{2}}\right\}
$$

satisfies $\left|\mathcal{U}^{\prime}\right| \ll X^{\frac{1}{2}-\nu+\min \left\{2 \sigma(\nu), \frac{\nu}{2}\right\}-\varepsilon}$. Then we have

$$
\int_{T_{0}}^{T}|G(1+i t)|^{2} d t \ll\left(\frac{T P_{1} \log X}{X}+1\right) \frac{1}{\log ^{2+\varepsilon} X} .
$$

Proof. Let $\alpha_{1}=100 \varepsilon$ and define $H=\log ^{10 \varepsilon} X$. Let

$$
Q_{v, H, 1}(s)=\sum_{e^{\frac{v}{H}} \leq p_{1}<e^{\frac{v+1}{H}}} p_{1}^{-s}, \quad Q_{v, H, 2}(s)=\sum_{e^{\frac{v}{H}} \leq p_{2}<e^{\frac{v+1}{H}}} p_{2}^{-s}
$$

and

$$
\begin{aligned}
& G_{v, H, 1}(s)= \sum_{\substack{p_{2} p_{3} p_{4} m \sim X e^{-v} \\
P_{2} \leq p_{2} \leq P_{2}^{1+\varepsilon} \\
p_{2}<p_{3}, p_{2} \leq p_{4} \\
\left(m, \mathcal{P}\left(p_{4}\right)\right)=1}} a_{p_{4} m}\left(p_{2} p_{3} p_{4} m\right)^{-s}, \\
& G_{v, H, 2}(s)=\sum_{\substack{p_{1} p_{3} p_{4} m \sim X e^{-\frac{v}{H}} \\
P_{1} \leq p_{1} \leq P_{1}^{1+\varepsilon} \\
\left(m, \mathcal{P}\left(p_{4}\right)\right)=1}} a_{p_{4} m}\left(p_{1} p_{3} p_{4} m\right)^{-s} .
\end{aligned}
$$

For $j=1,2$, we have

$$
\begin{aligned}
\int_{\mathcal{S}}|G(1+i t)|^{2} d t & \ll\left(\frac{T P_{1} \log X}{X}+1\right) \frac{1}{\log ^{2+\varepsilon} X} \\
& +H^{2}\left(\log ^{2} P_{j}\right)\left(\log ^{10(j-1)} X\right) \int_{\mathcal{S}}\left|Q_{v_{j}, H, j}(1+i t) G_{v_{j}, H, j}(1+i t)\right|^{2} d t
\end{aligned}
$$


for some $v_{j} \in\left[H \log P_{j},(1+\varepsilon) H \log P_{j}\right]$ and any measurable $\mathcal{S} \subset\left[T_{0}, T\right]$. In the case $j=1$, this follows from Lemmas 2 and 11, while in the case $j=2$, we use Perron's formula to separate the variables in $G(s)$. We partition $\left[T_{0}, T\right]$ as $\mathcal{T}_{1} \cup \mathcal{T}_{2} \cup \mathcal{T}$ with

$$
\begin{aligned}
& \mathcal{T}_{1}=\left\{t \in\left[T_{0}, T\right]:\left|Q_{v_{1}, H, 1}(1+i t)\right| \leq P_{1}^{-\alpha_{1}}\right\}, \\
& \mathcal{T}_{2}=\left\{t \in\left[T_{0}, T\right]:\left|Q_{v_{2}, H, 2}(1+i t)\right| \leq P_{2}^{-\alpha_{2}}\right\} \backslash \mathcal{T}_{1},
\end{aligned}
$$

and $\mathcal{T}=\left[T_{0}, T\right] \backslash\left(\mathcal{T}_{1} \cup \mathcal{T}_{2}\right)$.

What remains to be done is estimating the integrals

$$
B_{j}=H^{2}\left(\log ^{2} P_{j}\right)\left(\log ^{10(j-1)} X\right) \int_{\mathcal{T}_{j}}\left|Q_{v_{j}, H, j}(1+i t) G_{v_{j}, H, j}(1+i t)\right|^{2} d t
$$

for $j=1,2$, as well as

$$
B=H^{2}\left(\log ^{10} X\right) \int_{\mathcal{T}}\left|Q_{v_{2}, H, 2}(1+i t) G_{v_{2}, H, 2}(1+i t)\right|^{2} d t .
$$

We have $B_{1} \ll\left(\frac{T P_{1} \log X}{X}+1\right) \frac{P_{1}^{10 \varepsilon-\alpha_{1}}}{\log ^{2} X}$ by Proposition 1 and Lemma 11 , and this is small enough since $\alpha_{1}=100 \varepsilon$. We also have, by Proposition 22 with $\ell=\left\lceil\frac{\log P_{2}}{\log P_{1}}\right\rceil$,

$$
\begin{aligned}
B_{2} & \ll H^{2}\left(\log ^{20} X\right) P_{2}^{-2 \alpha_{2}} P_{1}^{(2+10 \varepsilon) \alpha_{1} \ell} \ell^{(1+o(1)) \ell} \\
& \ll P_{2}^{2\left(\alpha_{1}-\alpha_{2}\right)+20 \varepsilon+\frac{1+2 \varepsilon}{a}} \\
& \ll P_{2}^{-\varepsilon} \ll(\log X)^{-100},
\end{aligned}
$$

as long as $a \geq \frac{1}{2\left(\alpha_{2}-\alpha_{1}\right)}+\frac{C_{2}}{2} \varepsilon$, say. Lastly, Proposition 5 gives, for some well-spaced $\mathcal{U}^{\prime}$ of the type mentioned in the proposition,

$$
B \ll(\log X)^{-50}+X^{\frac{1}{2}-\min \left\{2 \sigma(\nu), \frac{\nu}{2}\right\}+o(1)} \frac{\left|\mathcal{U}^{\prime}\right| X^{\nu+o(1)}}{X} \ll(\log X)^{-50}
$$

by our assumption on $\mathcal{U}^{\prime}$. Now the proof is complete.

\subsection{Cases of $\Sigma_{1}(h)$ and $\Sigma_{2}(h)$}

Let $\lambda_{d}^{+}$and $\lambda_{d}^{-}$be the sieve weights of Brun's pure sieve with $R=2\left\lfloor(\log \log X)^{\frac{3}{2}}\right\rfloor$ and sieving parameter $w=\exp \left(\frac{\log X}{(\log \log X)^{3}}\right)$. We have

$$
\sum_{\substack{x \leq p_{1} d n \leq x+h \\ P_{1} \leq p_{1} \leq P_{1}^{1+\varepsilon}}} \lambda_{d}^{-} \leq \sum_{\substack{\frac{x}{p_{1}} \leq n \leq \frac{x+h}{p_{1}} \\ P_{1} \leq p_{1} \leq P_{1}^{1+\varepsilon} \\(n, \mathcal{P}(w))=1 \\ n>1}} 1=\Sigma_{1}(h) \leq \sum_{\substack{x \leq p_{1} d n \leq x+h \\ P_{1} \leq p_{1} \leq P_{1}^{1+\varepsilon}}} \lambda_{d}^{+} .
$$


We consider the lower bound; the upper bound can be considered similarly. Letting $X_{1}=\frac{X}{T_{0}^{3}}$ with $T_{0}=X^{0.01}$, we have

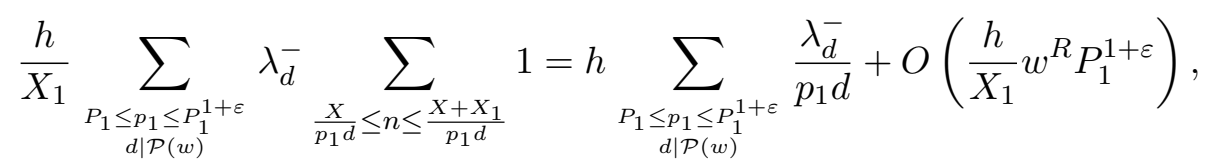

so

$$
\begin{aligned}
\Sigma_{1}(h) \geq & \sum_{\substack{d \mid \mathcal{P}(w) \\
P_{1} \leq p_{1} \leq P_{1}^{1+\varepsilon}}} \lambda_{d}^{-} \frac{h}{p_{1} d}+\left(\sum_{\substack{x \leq p_{1} d n \leq x+h \\
P_{1} \leq p_{1} \leq P_{1}^{1+\varepsilon}}} \lambda_{d}^{-}-\frac{h}{X_{1}} \sum_{\substack{X \leq p_{1} d n \leq X+X_{1} \\
P_{1} \leq p_{1} \leq P_{1}^{1+\varepsilon}}} \lambda_{d}^{-}\right) \\
& +O\left(\frac{1}{\log ^{100} X}\right) .
\end{aligned}
$$

By the fundamental lemma of the sieve (see e.g. [4, Chapter 6]), we further deduce that

$$
\begin{aligned}
\sum_{\substack{d \mid \mathcal{P}(w) \\
P_{1} \leq p_{1} \leq P_{1}^{1+\varepsilon}}} \lambda_{d}^{-} \frac{h}{p_{1} d} & =\left(1+O\left((\log X)^{-100}\right)\right) \sum_{\substack{d \mid \mathcal{P}(w) \\
P_{1} \leq p_{1} \leq P_{1}^{1+\varepsilon}}} \lambda_{d}^{+} \frac{h}{p_{1} d} \\
& \geq \frac{h}{X} \Sigma_{1}(X)+O\left(\frac{h}{\log ^{100} X}\right) .
\end{aligned}
$$

Therefore, we may concentrate on the expression in the parentheses in (17), which is a difference between a short and long average. By Lemma 1 , it is $o\left(\frac{h}{\log X}\right)$ for $h \geq P_{1} \log X$ and for almost all $x \leq X$, provided that

$$
\int_{T_{0}}^{T}|F(1+i t)|^{2} d t=o\left(\left(\frac{T P_{1} \log X}{X}+1\right) \frac{1}{\log ^{2} X}\right)
$$

for all $T \geq T_{0}$, where $T_{0}=X^{0.01}$, and

$$
F(s)=\sum_{\substack{p_{1} d n \sim X \\ P_{1} \leq p_{1} \leq P_{1}^{1+\varepsilon}}} \lambda_{d}^{-}\left(p_{1} d n\right)^{-s} .
$$

Such an estimate is given by the following proposition, which is invoked again in the case of the sum $\Sigma_{2}(h)$.

Proposition 8. Let $\varepsilon>0, P_{1}=\log ^{a} X$ with $a \geq 2+C_{3} \varepsilon$ and

$$
F(s)=\sum_{\substack{p_{1} d n \sim X \\ P_{1} \leq p_{1} \leq P_{1}^{1+\varepsilon}}} \lambda_{d}^{ \pm}\left(p_{1} d n\right)^{-s} \quad \text { or } \quad F(s)=\sum_{\substack{p_{1} p d n \sim X \\ P_{1} \leq p_{1} \leq P_{1}^{1+\varepsilon} \\ M \leq p \leq M^{1+\varepsilon}}} \lambda_{d}^{ \pm}\left(p_{1} p d n\right)^{-s}
$$

with $M \ll X^{\frac{1}{2}+o(1)}, X^{1+o(1)} \gg T \geq T_{0}=X^{0.01}$ as before, and either + or - sign chosen throughout. Then,

$$
\int_{T_{0}}^{T}|F(1+i t)|^{2} d t \ll\left(\frac{T P_{1} \log X}{X}+1\right) \frac{1}{\log ^{2+\varepsilon} X} .
$$


Proof. Let $D$ be a large constant, and for positive integer $v$ and $H=\log ^{10 \varepsilon} X$ denote

$$
P_{v, H}(s)=\sum_{e^{\frac{v}{H}} \leq p<e^{\frac{v+1}{H}}} p^{-s}
$$

and

$$
F_{v, H}(s)=\sum_{d n \sim X e^{-\frac{v}{H}}} \lambda_{d}^{ \pm}(d n)^{-s} \quad \text { or } \quad F_{v, H}(s)=\sum_{\substack{p d n \sim X e^{-\frac{v}{H}} \\ M \leq p \leq M^{1+\varepsilon}}} \lambda_{d}^{ \pm}(p d n)^{-s} .
$$

Lemma 2 gives

$$
\begin{aligned}
& \int_{T_{0}}^{T}|F(1+i t)|^{2} d t \ll H^{2}(\log \log X)^{2} \int_{T_{0}}^{T}\left|P_{v_{0}, H}(1+i t) F_{v_{0}, H}(1+i t)\right|^{2} d t
\end{aligned}
$$

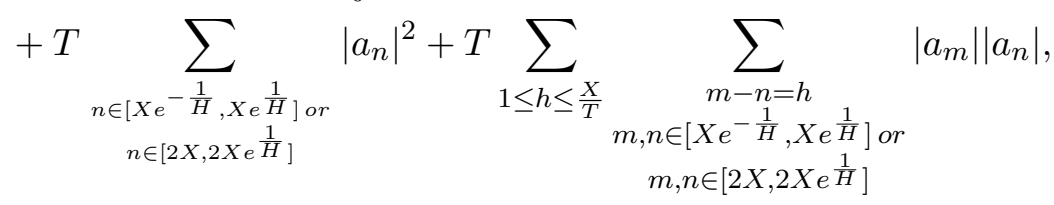

for some $v_{0} \in I_{0}$, where $I_{0}=\left[H \log P_{1}, H \log P_{1}^{1+\varepsilon}\right]$ and

$$
a_{m}=\sum_{\substack{p_{1} \mid m \\ P_{1} \leq p_{1} \leq P_{1}^{1+\varepsilon}}}\left|\sum_{m=p_{1} d n} \lambda_{d}^{ \pm}\right| \quad \text { or } a_{m}=\sum_{\substack{p_{1} \mid m \\ P_{1} \leq p_{1} \leq P_{1}^{1+\varepsilon}}}\left|\sum_{\substack{m=p_{1} p d n \\ M \leq p \leq M^{1+\varepsilon}}} \lambda_{d}^{ \pm}\right|
$$

Lemma 13 tells that the last two terms in 18 contribute, for some constant $C>0$,

$$
\begin{aligned}
& \ll \frac{T}{X}\left(\frac{(\log \log X)^{C}}{H} \cdot \frac{1}{\log X}+\frac{(\log \log X)^{C}}{H} \cdot \frac{X}{T} \cdot \frac{1}{\log ^{2} X}\right) \\
& \ll\left(\frac{T P_{1} \log X}{X}+1\right) \cdot \frac{1}{\log ^{2+\varepsilon} X}
\end{aligned}
$$

by the definition of $H$. We are now left with estimating the integral in (18). We consider the integrals in two parts, namely the part over $\mathcal{T}_{1}$ and its complement, with

$$
\mathcal{T}_{1}=\left\{t \in\left[T_{0}, T\right]:\left|P_{v_{0}, H}(1+i t)\right| \leq P_{1}^{-100 \varepsilon}\right\} .
$$

The case of $\mathcal{T}_{1}$ is dealt with Proposition 1 and Lemma 13 , and it contributes

$$
\begin{aligned}
& \ll H^{2}(\log \log X)^{2} \frac{T}{X} P_{1}^{1-200 \varepsilon}\left(S_{1}\left(\frac{X}{P_{1}},\left(a_{n}\right)\right)+S_{2}\left(\frac{X}{P_{1}},\left(a_{n}\right)\right)\right) \\
& \ll(\log \log X)^{C}\left(\frac{T}{X} \cdot \frac{1}{\log X}+\frac{1}{P_{1}} \cdot \frac{1}{\log ^{2} X}\right) \cdot P_{1}^{1-100 \varepsilon} \\
& \ll\left(\frac{T P_{1} \log X}{X}+1\right) \cdot \frac{1}{\log ^{2+\varepsilon} X},
\end{aligned}
$$


where the coefficients $a_{n}$ involved in definition of $S_{i}\left(X,\left(a_{n}\right)\right)$ are given by $(19)$.

We turn to the integral over the complement of $\mathcal{T}_{1}$ and resort to the Watt-type Proposition 6. Let $\ell$ be a large positive integer such that $P_{1}^{\ell}=X^{\varepsilon+o(1)}$. Letting $N_{a}(s)=\sum_{n \sim X e^{-a}} n^{-s}$ and

$$
M_{v, H}(s)=\sum_{\substack{\frac{v}{H} \leq p_{1} d<e^{\frac{v+1}{H}} \\ P_{1} \leq p_{1} \leq P_{1}^{1+\varepsilon}}} \lambda_{d}^{ \pm}\left(p_{1} d\right)^{-s} \quad \text { or } \quad M_{v, H}(s)=\sum_{\substack{\frac{v}{e^{H}} \leq p_{1} p d<e^{\frac{v+1}{H}} \\ P_{1} \leq p_{1} \leq P_{1}^{1+\varepsilon} \\ M \leq p \leq M^{1+\varepsilon}}} \lambda_{d}^{ \pm}\left(p_{1} p d\right)^{-s},
$$

an application of Perron's formula to separate variables, along with Lemma 13 and $\mid P_{v_{1}, H}(1+$ it) $\left.P_{1}^{100 \varepsilon}\right|^{2 \ell} \geq 1$, yields

$$
\begin{aligned}
& \int_{\left[T_{0}, T\right] \backslash \mathcal{T}_{1}}|F(1+i t)|^{2} d t \\
& \ll H^{2}\left(\log ^{10} X\right) P_{1}^{200 \varepsilon \ell} \int_{T_{0}}^{T}\left|P_{v_{0}, H}(1+i t)^{\ell} M_{v_{1}, H}(1+i t) N_{\frac{v_{1}}{H}}(1+i t)\right|^{2} d t \\
& +\left(\frac{T P_{1} \log X}{X}+1\right) \cdot \frac{1}{\log ^{2+\varepsilon} X}
\end{aligned}
$$

for some $v_{1} \in I_{1}$, where $I_{1}=\left[H \log M, H \log \left(M^{1+\varepsilon} w^{R}\right)\right]$. Now Proposition 6 with $N(s)=$ $N_{\frac{v_{1}}{H}}(s), M(s)=M_{v_{1}, H}(s), P(s) \equiv 1, Q(s)=P_{v_{0}, H}(s)^{\ell}$ and $\ell=\left\lfloor\frac{\varepsilon \log X}{\log P_{1}}\right\rfloor$ bounds 20 with

$$
X^{o(1)} P_{1}^{200 \varepsilon \ell}\left(Q^{-1}+\frac{1}{T_{0}}\right)(\ell !)^{2} \ll\left(P_{1}^{-1}\left(\log ^{2} X\right)\right)^{(1+o(1)) \ell}+X^{-\varepsilon} \ll X^{-\varepsilon^{2}}
$$

for $a \geq 2+C_{3} \varepsilon$, since the condition $M^{2} P \ll X^{1+o(1)}$ certainly holds.

Note that Proposition 8 immediately shows that

$$
\frac{1}{h} \Sigma_{1}(h)-\frac{1}{X_{1}} \Sigma_{1}\left(X_{1}\right) \geq o\left(\frac{1}{\log X}\right)
$$

for almost all $x \leq X$, where $X_{1}=\frac{X}{T_{0}^{3}}$. Taking into account formula (17) and repeating the above argument with lower bound sieve weights replaced with upper bound sieve weights, we see that the reverse inequality holds, so $\frac{1}{h} \Sigma_{1}(h)$ can be replaced with its dyadic counterpart $\frac{1}{X} \Sigma_{1}(X)$ almost always.

Now we deal with $\Sigma_{2}(h)$. We use the same strategy, so that for example for the lower bound we start with

$$
\Sigma_{2} \geq \sum_{\substack{x \leq p_{1} p d n \leq x+h \\ P_{1} \leq p_{1} \leq P_{1}^{1+\varepsilon}}} \lambda_{d}^{-},
$$


an inequality that is valid even when the interval $\left[\frac{x}{p_{1} p}, \frac{x+h}{p_{1} p}\right]$ contains no integers. This leads us to study the Dirichlet polynomial

$$
F^{*}(s)=\sum_{\substack{p_{1} p d n \sim X \\ P_{1} \leq p_{1} \leq P_{1}^{1+\varepsilon} \\ w \leq p<\sqrt{x}}} \lambda_{d}^{-}\left(p_{1} p d n\right)^{-s},
$$

where the variable $p$ can be divided into $\ll \log \log X$ intervals of the form $\left[M, M^{1+\varepsilon}\right]$ with $M \ll X^{\frac{1}{2}+o(1)}$ (the value of $\varepsilon$ may be varied so that the division becomes exact). For each of these Dirichlet polynomials where $p$ is restricted, Proposition 8 gives a bound of $\left(\frac{T P_{1} \log X}{X}+1\right)(\log X)^{-2-\varepsilon}$ for their second moment. Now by the same argument as for $\Sigma_{1}(h)$, we infer that $\frac{1}{h} \Sigma_{2}(h)$ can also be replaced with its dyadic counterpart $\frac{1}{X} \Sigma_{2}(X)$ almost always.

\subsection{Case of $\Sigma_{3}(h)$}

We are left with the sum $\Sigma_{3}(h)$. This is the case that determines which value of $a$ we obtain (and hence the value of $c$, which is just $a+1$ ), since so far in all cases $a \geq 2+C_{4} \varepsilon$ has been a sufficient assumption. We will establish the value $a=2.51$.

Let $\beta_{1}, \beta_{2}, \beta \in\left(\frac{1}{6}, \frac{1}{2}\right)$ be parameters which are given the values

$$
\beta_{1}=0.1680, \quad \beta_{2}=0.1803, \quad \beta=0.1950
$$

to optimize various subsequent conditions. We split $\Sigma_{3}(h)$ into three parts $\Sigma_{3}^{(1)}(h), \Sigma_{3}^{(2)}(h)$ and $\Sigma_{3}^{(3)}(h)$, say, the first sum being a type II sum that can be evaluated asymptotically, the second being a type I sum (after Buchstab's identity) that can mostly be evaluated asymptotically, and the third being a type II sum that can be transformed into Buchstab integrals whose value is suitably small. Explicitly, let

$$
\Sigma_{3}^{(i)}(h)=\sum_{\substack{x \leq p_{1} q_{1} q_{2} n \leq x+h \\ P_{1} \leq p_{1} \leq P_{1}^{1+\varepsilon} \\\left(q_{1}, q_{2}\right) \in A_{i} \\\left(n, \mathcal{P}\left(q_{2}\right)\right)=1 \\ n>1}} 1, \quad i=1,2,3
$$

with

$$
\begin{aligned}
& A_{1}=\left\{\left(q_{1}, q_{2}\right): w \leq q_{2}<q_{1}, \text { one of } q_{1}, q_{2} \in\left[w, X^{\beta_{1}}\right] \cup\left[X^{\beta_{2}}, X^{\beta}\right]\right\}, \\
& A_{2}=\left\{\left(q_{1}, q_{2}\right): w \leq q_{2}<q_{1}, \text { either } q_{1}^{2} q_{2}^{3} \leq X \text { or } q_{1} q_{2}^{4} \leq X, q_{1} \leq X^{\frac{1}{4}-2 \varepsilon}\right\} \backslash A_{1} \\
& A_{3}=\left\{\left(q_{1}, q_{2}\right): w \leq q_{2}<q_{1} \leq X^{\frac{1}{2}}\right\} \backslash\left(A_{1} \cup A_{2}\right) .
\end{aligned}
$$

The underlying idea is that the small variable in $A_{1}$ enables efficient use of large values theorems, the conditions in $A_{2}$ make it possible to apply Watt's theorem (after two applications of Buchstab's identity), and the remaining set $A_{3}$ can be shown to contribute not too much. We study the sums $\Sigma_{3}^{(i)}(h)$ separately, starting with $\Sigma_{3}^{(1)}(h)$. 


\subsubsection{Type II sums}

We consider the Type II sum $\Sigma_{3}^{(1)}(h)$. In order to prove that $\frac{1}{h} \Sigma_{3}^{(1)}(h)$ is asymptotically $\frac{1}{X} \Sigma_{3}^{(1)}(X)$ almost always, it suffices to prove that $\frac{1}{h} \Sigma_{3}^{(1)}(h)$ is asymptotically $\frac{1}{X_{1}} \Sigma_{3}^{(1)}\left(X_{1}\right)$ almost always with $X_{1}=\frac{X}{T_{0}^{3}}$, and then apply the prime number theorem in short intervals. For this latter asymptotic equivalence, it suffices to show that the Dirichlet polynomial

$$
G(s)=\sum_{\substack{p_{1} q_{1} q_{2} n \sim X \\ P_{1} \leq p_{1} \leq P_{1+\varepsilon}^{1+\varepsilon} \\ Q_{i} \leq q_{i} \leq P_{i}^{1+\varepsilon}, i \leq 2 \\ q_{2}<q_{1} \\\left(n, \mathcal{P}\left(q_{2}\right)\right)=1 \\ n>1}}\left(p_{1} q_{1} q_{2} n\right)^{-s}
$$

satisfies

$$
\int_{T_{0}}^{T}|G(1+i t)|^{2} d t \ll\left(\frac{T P_{1} \log X}{X}+1\right) \frac{1}{\log ^{2+\varepsilon} X}
$$

with $T \leq X^{1+o(1)}, T_{0}=X^{0.01}, P_{1}=\log ^{a} X$ and $Q_{1}, Q_{2} \geq w$ otherwise arbitrary, but either $Q_{1}$ or $Q_{2}$ is of size $X^{\nu+o(1)}$ with $\nu \in\left[0, \beta_{1}\right] \cup\left[\beta_{2}, \beta\right]$. These cases are similar, so assume $Q_{2}=X^{\nu+o(1)}$ with $\nu$ as above.

This is the setting of Proposition 7 . Therefore, if for every polynomial of the form

$$
M(s)=\sum_{m \sim M} \frac{b_{m}}{m^{s}},
$$

with $M=X^{\nu+o(1)}$ and $\left|b_{m}\right| \leq d_{r}(n)$ for fixed $r$, any well-spaced set

$$
\mathcal{U}^{\prime} \subset\left\{t \in[0, T]:|M(1+i t)| \geq M^{-\alpha_{2}}\right\}
$$

satisfies

$$
\left|\mathcal{U}^{\prime}\right| \ll X^{\frac{1}{2}-\nu+\min \left\{2 \sigma(\nu), \frac{\nu}{2}\right\}-\varepsilon},
$$

the $\operatorname{sum} \Sigma_{3}^{(1)}(h)$ has the anticipated asymptotic for $a \geq \frac{1}{2 \alpha_{2}}+C_{5} \varepsilon$. Of course, we fix $\alpha_{2}=\frac{1}{2 \cdot 2.51}+C_{6} \varepsilon$.

We are left with estimating $\left|\mathcal{U}^{\prime}\right|$, and to this end we utilize Jutila's large values theorem. Jutila's large values theorem (Lemma 7) applied to the $\ell$ th moment of $M(s)$ can be reformulated to say that if

$$
\mathcal{R}\left(\nu, \alpha_{2}, k, \ell\right)=\max \left\{2 \nu \alpha_{2} \ell,\left(6-\frac{2}{k}\right) \nu \alpha_{2} \ell+1-2 \nu \ell, 1+8 k \ell \nu \alpha_{2}-2 k \ell \nu\right\}
$$

and

$$
\overline{\mathcal{R}}\left(\nu, \alpha_{2}\right)=\min _{k, \ell \in\{1,2, \ldots\}} \mathcal{R}\left(\nu, \alpha_{2}, k, \ell\right),
$$


then $|\mathcal{U}| \ll X^{\tilde{\mathcal{R}}\left(\nu, \alpha_{2}\right)+o(1)}$. It turns out that the case $k=3$ is always optimal for us, and it suffices to restrict to $4 \leq \ell \leq 12$ (so our upper bound for $\overline{\mathcal{R}}\left(\nu, \alpha_{2}\right)$ is a minimum of 9 piecewise linear functions). Now we check that, with our choices of $\beta_{1}, \beta_{2}, \beta$ and $\alpha_{2}$,

$$
\overline{\mathcal{R}}\left(\nu, \alpha_{2}\right) \leq \frac{1}{2}-\nu+\min \left\{2 \sigma(\nu), \frac{\nu}{2}\right\}-\varepsilon
$$

for $\nu \in\left[0.05, \beta_{1}\right] \cup\left[\beta_{1}, \beta_{2}\right]$. Verifying this is straightforward, because both sides are piecewise linear functions 4

We must also prove the desired estimate for $\left|\mathcal{U}^{\prime}\right|$ in the range $\nu \in[0,0.05)$. In this case, we do not appeal to Jutila's large values theorem, but to Lemma 6 (along with its remark), which tells us that

$$
\left|\mathcal{U}^{\prime}\right| \ll T^{2 \alpha_{2}} X^{2 \alpha_{2} \nu+o(1)} \ll X^{0.42}<X^{\frac{1}{2}-\nu-\varepsilon}
$$

for the same value $\alpha_{2}=\frac{1}{2 \cdot 2.51}+C_{6} \varepsilon$. This means that for $c=3.51, \frac{1}{h} \Sigma_{3}^{(1)}(h)$ can be replaced with its dyadic counterpart almost always.

\subsubsection{Type I sums}

We turn to the sum $\Sigma_{3}^{(2)}(h)$. By applying Buchstab's identity twice, we find that

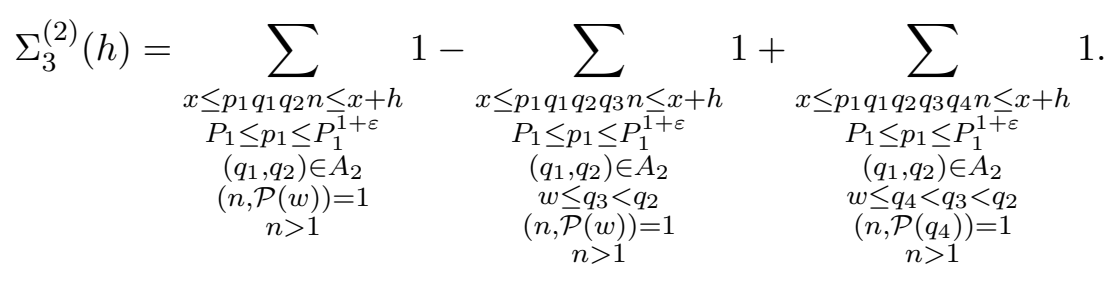

Call these sums $\Sigma_{3}^{(2,1)}(h), \Sigma_{3}^{(2,2)}(h)$ and $\Sigma_{3}^{(2,3)}(h)$, respectively. We show that $\frac{1}{h} \Sigma_{3}^{(2,1)}(h)$ and $\frac{1}{h} \Sigma_{3}^{(2,2)}(h)$ can be replaced with their dyadic counterparts almost always. We confine to studying $\Sigma_{3}^{(2,2)}(h)$, as $\Sigma_{3}^{(2,1)}(h)$ is easier to handle.

We may make in $\Sigma_{3}^{(2,2)}(h)$ the additional assumption that all the variables except $P_{1}$ are in the intervals $\left[X^{\beta_{1}}, X^{\beta_{2}}\right] \cup\left[X^{\beta}, X\right]$, since otherwise the sum can be dealt with in the same way as $\Sigma_{3}^{(1)}(h)$. We may also assume that $q_{i} \in\left[Q_{i}, Q_{i}^{1+\varepsilon}\right]$ for some $Q_{i}$. Defining

$$
F(s)=\sum_{\substack{p_{1} q_{1} q_{2} q_{3} d n \sim X \\ P_{1} \leq p_{1} \leq P_{1}^{1+\varepsilon} \\ Q_{i} \leq q_{i} \leq Q_{i}^{1+\varepsilon} \\\left(q_{1}, q_{2}\right) \in A}} \lambda_{d}^{ \pm}\left(p_{1} q_{1} q_{2} q_{3} d n\right)^{-s},
$$

\footnotetext{
${ }^{4}$ These computations can be carried out by hand with a bit of patience. For example, the case $\ell=4$ in Jutila's bound is good enough in the range $\nu \in\left[\frac{16315}{90496}, \frac{15311}{78512}\right]$, and the bound for $\ell=5$ is good enough when $\nu \in\left[\frac{753}{5554}, \frac{15311}{91112}\right]$. These intervals are $\left[\beta_{2}, \beta\right]$ and $\left[0.1356, \beta_{1}\right]$, up to rounding.
} 
with $\lambda_{d}^{ \pm}$the same Brun's sieve weights as before (the sign being the same throughout), and taking into account the prime number theorem in short intervals and Lemmas 1 and 2 , it suffices to show that

$$
\int_{T_{0}}^{T}|F(1+i t)|^{2} d t \ll\left(\frac{T P_{1} \log X}{X}+1\right) \frac{1}{\log ^{2+\varepsilon} X} .
$$

This bound is achieved similarly as in Proposition 8. Indeed, if $\mathcal{T}_{1}$ is defined as in the proof of that proposition, the integral over $\mathcal{T}_{1}$ can be estimated in the same way as in that proposition. In the complementary case, we separate all the variables, and it remains to show that

$$
\begin{aligned}
& \int_{\left[T_{0}, T\right] \backslash \mathcal{T}_{1}}\left|P_{1}(1+i t) Q_{1}(1+i t) Q_{2}(1+i t) Q_{3}(1+i t) D(1+i t) N(1+i t)\right|^{2} d t \\
& \ll(\log X)^{-100},
\end{aligned}
$$

where $N(s)$ is a zeta sum, $P_{1}(s)$ and $Q_{i}(s)$ are polynomials supported on primes, and $D(s)$ has the sieve weights $\lambda_{d}$ as its coefficients (actually, $D(s)$ can be neglected by simply estimating it pointwise). Moreover, the lengths $P_{1}, Q_{i}, D$ and $N$ are from the same intervals as $p_{1}, q_{i}, d$ and $n$, respectively (in particular, $d \leq \exp \left(\frac{\log X}{\log \log X}\right)$ ). We appeal to Proposition 6 with $Q(s)=P_{1}(s)^{\ell}, P_{1}^{\ell}=X^{\varepsilon}$ and with $M(s)$ either $Q_{1}(s) Q_{3}(s)$ or $Q_{2}(s) Q_{3}(s)$. If $M(s)=Q_{1}(s) Q_{3}(s)$, the condition for Proposition 6 is $Q_{2} \leq X^{\frac{1}{4}-2 \varepsilon},\left(Q_{1} Q_{3}\right)^{2} Q_{2} \leq X$. If in turn $M(s)=Q_{2}(s) Q_{3}(s)$, the condition for Proposition 6 is $Q_{1} \leq X^{\frac{1}{4}-2 \varepsilon}, Q_{1}\left(Q_{2} Q_{3}\right)^{2} \leq X$, and one of these conditions is always satisfied in our domain $A_{2}$, since $Q_{3} \leq Q_{2}$ and automatically $Q_{2} \leq X^{\frac{1}{5}}$. Now it follows from (21) that for $a \geq 2+C_{7} \varepsilon, \Sigma_{3}^{(2,2)}(h)$ has the desired asymptotic, and $\Sigma_{3}^{(2,1)}(h)$ can be evaluated similarly.

In the sum $\Sigma_{3}^{(2,3)}(h)$, we may again assume that all the variables lie in the intervals $\left[X^{\beta_{1}}, X^{\beta_{2}}\right] \cup\left[X^{\beta}, X\right]$, as otherwise we can use the type II sum argument. Let $\Sigma_{3}^{(2,4)}(h)$ be what remains of $\Sigma_{3}^{(2,3)}(h)$ after this reduction. The sum $\Sigma_{3}^{(2,4)}$ results in a Buchstab integral, and hence is postponed to Subsection 6.3.3.

\subsubsection{Buchstab integrals}

We are left with the sums $\Sigma_{3}^{(3)}(h)$ and $\Sigma_{3}^{(2,4)}(h)$, for which no asymptotic was found. We want to show that

$$
\frac{1}{X} \Sigma_{3}^{(3)}(X)+\frac{1}{X} \Sigma_{3}^{(2,4)}(X) \leq(1-\varepsilon) \frac{1}{X} S_{X},
$$

which would complete the proof of Theorem 5, taking into account the estimates (15) and (16). The following lemma allows us to transform our sums into Buchstab integrals.

Lemma 16. Let a positive integer $k$ and $\eta>0$ be fixed. Let

$$
A \subset\left\{\left(u_{1}, \ldots, u_{k}\right) \in \mathbb{R}^{k}: u_{1}, \ldots, u_{k} \geq \eta, u_{1}+\ldots+u_{k} \leq 1-\eta\right\}
$$


be any set such that $1_{A}$ is Riemann integrable. For a point $q=\left(q_{1}, \ldots, q_{k}\right) \in \mathbb{R}^{k}$ and $X \geq 2$, define $\mathcal{L}(q)=\left(\frac{\log q_{1}}{\log X}, \ldots, \frac{\log q_{k}}{\log X}\right)$. Then

$$
\begin{aligned}
& \sum_{\substack{p_{1} q_{1} \cdots q_{k} n \sim X \\
P_{1} \leq p_{1} \leq P_{1}^{1+\varepsilon} \\
\mathcal{L}\left(q_{1}, \ldots, q_{k}\right) \in A \\
\left(n, \mathcal{P}\left(q_{k}\right)\right)=1}} 1 \\
& =(1+o(1)) \log (1+\varepsilon) \frac{X}{\log X} \int_{\left(u_{1}, \ldots, u_{k}\right) \in A} \omega\left(\frac{1-u_{1}-\cdots-u_{k}}{u_{k}}\right) \frac{d u}{u_{1} \cdots u_{k-1} u_{k}^{2}},
\end{aligned}
$$

where $\omega(\cdot)$ is Buchstab's function.

Proof. It suffices to prove the statement in the case that $A$ is a box, that is, a set of the form $I_{1} \times \ldots \times I_{k}$ with $I_{i}$ intervals. Indeed, if the statement holds for boxes, then it holds for finite unions of boxes. Moreover, since $1_{A}$ is Riemann integrable, for every $\delta>0$ there is a finite union $\mathcal{B}$ of boxes such that $A \backslash \mathcal{B}$ has measure at most $\delta$. The part of $A$ not contained in $\mathcal{B}$ contributes at most $\eta^{-k-1} \delta$ to the integral, and as $\delta \rightarrow 0$, this becomes arbitrarily small.

Now let $A$ be a box. Using the connection between Buchstab's function and the sieving function (see the Appendix of Harman's book [10]), summing partially, and using the change of variables $u_{i}=\frac{\log v_{i}}{\log X}$, we see that

$$
\begin{aligned}
\sum_{\substack{p_{1} q_{1} \cdots q_{k} n \sim X \\
P_{1} \leq p_{1} \leq P_{1}^{1+\varepsilon} \\
\mathcal{L}\left(q_{1}, \ldots, q_{k}\right) \in A \\
\left(n, \mathcal{P}\left(q_{k}\right)\right)=1}}=\sum_{\substack{P_{1} \leq p_{1} \leq P_{1}^{1+\varepsilon} \\
\mathcal{L}\left(q_{1}, \ldots, q_{k}\right) \in A}} S\left(\left[\frac{X}{p_{1} q_{1} \cdots q_{k}}, \frac{2 X}{p_{1} q_{1} \cdots q_{k}}\right], \mathbb{P}, q_{k}\right) \\
=(1+o(1)) \sum_{\substack{P_{1} \leq p_{1} \leq P_{1}^{1+\varepsilon} \\
\mathcal{L}\left(q_{1}, \ldots, q_{k}\right) \in A}} \frac{X}{p_{1} q_{1} \cdots q_{k} \log q_{k}} \omega\left(\frac{\log \frac{X}{p_{1} q_{1} \cdots q_{k}}}{\log q_{k}}\right) \\
=(1+o(1)) \sum_{P_{1} \leq p_{1} \leq P_{1}^{1+\varepsilon}} \frac{1}{p_{1}} \sum_{\mathcal{L}\left(q_{1}, \ldots, q_{k}\right) \in A} \frac{X}{q_{1} \cdots q_{k} \log q_{k}} \omega\left(\frac{\log \frac{X}{q_{1} \cdots q_{k}}}{\log q_{k}}\right) \\
=(b+o(1)) \int_{\mathcal{L}\left(v_{1}, \ldots, v_{k}\right) \in A} \frac{X}{v_{1} \cdots v_{k} \log v_{1} \cdots \log ^{2} v_{k}} \omega\left(\frac{\log \frac{X}{v_{1} \cdots v_{k}}}{\log v_{k}}\right) d v \\
=(b+o(1)) \frac{X}{\log X} \int_{\left(u_{1}, \ldots, u_{k}\right) \in A} \frac{1}{u_{1} \cdots u_{k}^{2}} \omega\left(\frac{1-u_{1}-\cdots u_{k}}{u_{k}}\right) d u
\end{aligned}
$$

with $b=\log (1+\varepsilon)$, as wanted.

Let

$$
\begin{aligned}
A_{3}^{*}= & \left\{\left(u_{1}, u_{2}\right): u_{2}<u_{1}, u_{1}, u_{2} \in\left[\beta_{1}, \beta_{2}\right] \cup\left[\beta, \frac{1}{2}\right], 2 u_{1}+3 u_{2} \geq 1,\right. \\
& \left.\max \left\{u_{1}+4 u_{2}, 4 u_{1}-10 \varepsilon\right\} \geq 1\right\}, \\
A_{2}^{*}= & \left\{\left(u_{1}, u_{2}, u_{3}, u_{4}\right): \beta_{1} \leq u_{4}<u_{3}<u_{2}<u_{1}, u_{1}, u_{2}, u_{3}, u_{4} \notin\left[\beta_{2}, \beta\right],\left(u_{1}, u_{2}\right) \in A_{2}\right\}
\end{aligned}
$$


be the sets corresponding to the summation conditions in $\Sigma_{3}^{(3)}(X)$ and $\Sigma_{3}^{(2,4)}(X)$, respectively. The lemma above directly implies that

$$
\begin{aligned}
\frac{1}{X} \Sigma_{3}^{(3)}(X) & =\frac{(1+o(1)) \log (1+\varepsilon)}{\log X} J_{1}, \\
\frac{1}{X} \Sigma_{3}^{(2,4)}(X) & =\frac{(1+o(1)) \log (1+\varepsilon)}{\log X} J_{2}, \\
\frac{1}{X} S_{X} & =\frac{(1+o(1)) \log (1+\varepsilon)}{\log X}
\end{aligned}
$$

where $J_{1}$ and $J_{2}$ are given by

$$
\begin{aligned}
& J_{1}=\int_{\left(u_{1}, u_{2}\right) \in A_{3}^{*}} \omega\left(\frac{1-u_{1}-u_{2}}{u_{2}}\right) \frac{d u}{u_{1} u_{2}^{2}}, \\
& J_{2}=\int_{\left(u_{1}, u_{2}, u_{3}, u_{4}\right) \in A_{2}^{*}} \omega\left(\frac{1-u_{1}-u_{2}-u_{3}-u_{4}}{u_{4}}\right) \frac{d u}{u_{1} u_{2} u_{3} u_{4}^{2}} .
\end{aligned}
$$

To compute $J_{1}$, we approximate Buchstab's function by

$$
\omega(u) \leq\left\{\begin{array}{l}
0, \quad u<1 \\
\frac{1}{u}, \quad 1 \leq u \leq 2 \\
\frac{1+\log (u-1)}{u}, \quad 2 \leq u \leq 3 \\
\frac{1+\log 2}{3}, \quad u>3
\end{array}\right.
$$

For $u \leq 3$ this is an equality, and for $u>3$ the bound very sharp (it differs from the limiting value $e^{-\gamma}$, where $\gamma$ is Euler's constant, by less than 0.003 ), but we only need the fact that it is an upper bound. We compute with Mathematica that $J_{1}<0.988$ (when $\varepsilon$ in the definition of $A_{3}^{*}$ is small enough) $!^{5}$ The integral $J_{2}$ only gives a minor contribution, and hence can be estimated crudely as

$$
\begin{aligned}
J_{2} & \leq \beta_{1}^{-5} \int_{\substack{\left(u_{1}, u_{2}, u_{3}, u_{4}\right) \in A_{2}^{*} \\
u_{1}+u_{2}+u_{3}+2 u_{4} \leq 1}} d u \\
& <\beta_{1}^{-5} \int_{\substack{\beta_{1}<u_{4}<u_{3}<u_{2}<u_{1} \\
u_{1}+u_{2}+u_{3}+2 u_{4} \leq 1}} d u<0.007
\end{aligned}
$$

with Mathematica (the last integral could actually be evaluated exactly). To sum up, we have $J_{1}+J_{2}<0.995<1-\varepsilon$, and this means, in view of $(16)$, that with our parameter choices $\beta_{1}, \beta_{2}, \beta$, the sums $\Sigma_{3}^{(3)}(X)$ and $\Sigma_{3}^{(2,4)}(X)$ can be discarded. Now, from (15) and (16) we have $\frac{1}{h} S_{h}(x) \geq \varepsilon \cdot \frac{1}{X} S_{X}$, so Theorem 5 is proved.

\footnotetext{
${ }^{5}$ The Mathematica code can be found at http://codepad.org/XCqx2iH3. There is also a Python code for computing the integral at http://codepad.org/cVx065z5, where the integration method is a rigorous computation of an upper Riemann sum.
} 
Remark 10. We can now observe that $c=3+\varepsilon$ is the limit of this method. Indeed, we are forced to take $\alpha_{2} \leq \frac{1}{4}$ in the type II case, because nothing nontrivial is known about the large values of Dirichlet polynomials beyond this region, and consequently $a=\frac{1}{2 \alpha_{2}}+\varepsilon \geq 2+\varepsilon$ and $c \geq 3+\varepsilon$.

\section{REFERENCES}

[1] R. C. Baker, G. Harman, and J. Pintz. The difference between consecutive primes. II. Proc. London Math. Soc. (3), 83(3):532-562, 2001.

[2] J. Bourgain. On large values estimates for Dirichlet polynomials and the density hypothesis for the Riemann zeta function. Internat. Math. Res. Notices, (3):133-146, 2000 .

[3] T. Freiberg. Short intervals with a given number of primes. J. Number Theory, 163:159-171, 2016.

[4] J. Friedlander and H. Iwaniec. Opera de cribro, volume 57 of American Mathematical Society Colloquium Publications. American Mathematical Society, Providence, RI, 2010 .

[5] P. X. Gallagher. On the distribution of primes in short intervals. Mathematika, 23(1):4-9, 1976.

[6] D. A. Goldston, J. Pintz, and C. Y. Ylldırım. Positive proportion of small gaps between consecutive primes. Publ. Math. Debrecen, 79(3-4):433-444, 2011.

[7] D. A. Goldston, J. Pintz, and C. Y. Yıldırım. Primes in tuples IV: Density of small gaps between consecutive primes. Acta Arith., 160(1):37-53, 2013.

[8] G. H. Hardy and S. Ramanujan. The normal number of prime factors of a number $n$ [Quart. J. Math. 48 (1917), 76-92]. In Collected papers of Srinivasa Ramanujan, pages 262-275. AMS Chelsea Publ., Providence, RI, 2000.

[9] G. Harman. Almost-primes in short intervals. Math. Ann., 258(1):107-112, 1981/82.

[10] G. Harman. Prime-detecting sieves, volume 33 of London Mathematical Society Monographs Series. Princeton University Press, Princeton, NJ, 2007.

[11] D. R. Heath-Brown. Prime numbers in short intervals and a generalized Vaughan identity. Canad. J. Math., 34(6):1365-1377, 1982.

[12] H. Iwaniec and E. Kowalski. Analytic number theory, volume 53 of American Mathematical Society Colloquium Publications. American Mathematical Society, Providence, RI, 2004.

[13] C. Jia. Almost all short intervals containing prime numbers. Acta Arith., 76(1):21-84, 1996.

[14] M. Jutila. Zero-density estimates for L-functions. Acta Arith., 32(1):55-62, 1977. 
[15] K. Matomäki and M. Radziwiłł. Multiplicative functions in short intervals. To appear in Ann. of Math.

[16] H. Mikawa. Almost-primes in arithmetic progressions and short intervals. Tsukuba J. Math., 13(2):387-401, 1989.

[17] H. L. Montgomery. Ten lectures on the interface between analytic number theory and harmonic analysis, volume 84 of CBMS Regional Conference Series in Mathematics. Published for the Conference Board of the Mathematical Sciences, Washington, DC; by the American Mathematical Society, Providence, RI, 1994.

[18] A. Selberg. On the normal density of primes in small intervals, and the difference between consecutive primes. Arch. Math. Naturvid., 47(6):87-105, 1943.

[19] N. Watt. Kloosterman sums and a mean value for Dirichlet polynomials. J. Number Theory, 53(1):179-210, 1995.

[20] N. Watt. Short intervals almost all containing primes. Acta Arith., 72(2):131-167, 1995.

[21] D. Wolke. Fast-Primzahlen in kurzen Intervallen. Math. Ann., 244(3):233-242, 1979.

Department of Mathematics and statistics, University of Turku, 20014 Turku, FINLAND

Email address: joni.p.teravainen@utu.fi 